\title{
Generalizações e teoremas limites para modelos estocásticos de rumores
}

\author{
Pablo Martín Rodríguez \\ TESE APRESENTADA \\ $\mathrm{AO}$ \\ Instituto de MATEMÁticA E Estatística \\ DA \\ Universidade DE SÃo PAUlo \\ PARA \\ OBTENÇÃO DO TÍTULO \\ $\mathrm{DE}$ \\ DOUTOR EM CIÊNCIAS \\ Programa: Estatística \\ Orientador: Prof. Dr. Fabio Prates Machado \\ Co-orientador: Prof. Dr. Élcio Lebensztayn
}

Durante o desenvolvimento deste trabalho o autor recebeu auxílio financeiro da FAPESP

São Paulo, 10 de novembro de 2010 


\title{
Generalizações e teoremas limites para modelos estocásticos de rumores
}

\author{
Esta versão definitiva da tese \\ contém as correções e alterações sugeridas pela \\ Comissão Julgadora durante a defesa realizada \\ por Pablo Martín Rodríguez em 13/10/2010.
}

Comissão Julgadora:

- Prof. Dr. Fábio Prates Machado (orientador) - IME - USP

- Prof. Dr. Élcio Lebensztayn (co-orientador) - IME - USP

- Profa. Dra. Nancy Lopes Garcia - UNICAMP

- Prof. Dr. Sebastian Pablo Grynberg - UBA

- Prof. Dr. Pablo José Groisman - UBA 
Aos meus pais Victor e Mary

Aos meus irmãos Victoria, Emanuel e Lucía

Às minhas queridas Paula e Luisa

À memória de Miriam Elizabeth Pacheco, professora e amiga da Universidad Nacional de la Patagonia, que indicou esse caminho para minha vida. 


\section{Agradecimentos}

Aos meus orientadores Fábio Machado e Élcio Lebenstzayn pela confiança, apoio e orientação durante esses anos. Sem eles este trabalho não teria sido possível.

Ao grupo de probabilidade do Instituto de Matemática e Estatística da Universidade de São Paulo pelas ótimas condições para a realização desta pesquisa. Em especial aos professores Luiz Renato Fontes, Pablo Ferrari, Serguei Popov e Vladimir Belitsky por seus ensinamentos.

A todos os colegas e amigos que, direta ou indiretamente, contribuíram para o enriquecimento deste trabalho. Pelas tão variadas quanto interessantes discussões agradeço a Cristian Coletti, Christophe Gallesco, Alexsandro Gallo, Renato Gava, Rafael Grisi, Fabio Marcellus, Mauricio Martinez, Bernardo Marques, Valdivino Vargas, Natalia Viana, Sebastián Vidal e Sandra Zapata. Em especial a Paula Cadavid por suas críticas e sugestões desde o começo deste processo.

Às pessoas das diferentes instituições que tive oportunidade de visitar. Em particular, merecem meu especial agradecimento os professores Rahul Roy, Anish Sarkar e Antar Bandyopadhyay pela hospitalidade e atenção dispensada na minha visita ao Indian Satistical Institute, Delhi Centre. Pelos mesmos motivos, na minha visita à Universidad de Buenos Aires, agradeço especialmente a Nahuel Soprano, Sergio López e aos professores Pablo Groisman e Inés Armendáriz.

À comissão julgadora pelas valiosas críticas e correções que ajudaram a melhorar esta versão final da tese.

À FAPESP (Processo 2006/04524-0), pelo apoio financeiro. 


\section{Resumo}

Os modelos de Daley-Kendall e Maki-Thompson são os dois modelos estocásticos para difusão de rumores mais citados até o momento. Em ambos, uma população finita fechada e totalmente misturada é subdividida em três classes de indivíduos denominados ignorantes, informantes e contidos. Depois de um rumor ser introduzido na população, difunde-se através desta seguindo determinadas regras que dependem da classe à qual a pessoa que sabe do rumor pertence. Tanto a proporção final de indivíduos que nunca chegam a conhecer o rumor quanto o tempo que este demora em ser difundido são variáveis de interesse para os modelos propostos.

As técnicas encontradas na literatura para estudar modelos de rumores são o princípio de difusão de constantes arbitrárias; argumentos de martingais; o método de funções geradoras e a análise de versões determinísticas do processo. Neste trabalho apresentamos uma alternativa para essas técnicas baseando-nos na teoria de cadeias de Markov "density dependent". O uso desta nova abordagem nos permite apresentar resultados assintóticos para um modelo geral que tem como casos particulares os famosos modelos de Daley-Kendall e Maki-Thompson, além de variações de modelos de rumores apresentados na literatura recentemente.

Palavras-chave: rumores estocásticos, modelo de Daley-Kendall, modelo de Maki-Thompson, teoremas limites, cadeias de Markov "density dependent". 


\section{Abstract}

Daley-Kendall and Maki-Thompson models are the two most cited stochastic models for the spread of rumours phenomena, in scientific literature. In both, a closed homogeneously mixing population is subdivided into three classes of individuals called ignorants, spreaders and stiflers. After a rumor is introduced in the population, it spreads by following certain rules that depend on the class to which the individual who knows the rumor belongs. Both the final proportion of the population never hearing the rumor and the time it takes are variables of interest for the proposed models.

The main tools used to study stochastic rumours have been the principle of the diffusion of arbitrary constants, martingale arguments, generating functions and the study of analogue deterministic versions. Relying on the theory of density dependent Markov chains, we present an alternative to these tools. This approach allows us to establish asymptotical results for a general model that has as particular cases the classical Daley-Kendall and Maki-Thompson models, and other variations for rumour models reported in the literature recently.

Keywords: stochastic rumours, Daley-Kendall model, Maki-Thompson model, limit theorems, density dependent Markov chains. 


\section{Sumário}

Lista de Símbolos $\quad$ vii

1 Introdução 1

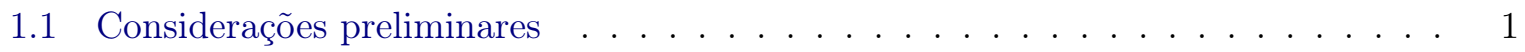

1.2 Epidemias e rumores . . . . . . . . . . . . . . . . . . . . . . . 2

1.3 O modelo de Daley-Kendall . . . . . . . . . . . . . . . . . . . . . . 4

$1.4 \mathrm{O}$ modelo de Maki-Thompson . . . . . . . . . . . . . . . . 6

2 Generalização do modelo de Maki-Thompson $\quad 7$

2.1 O modelo . . . . . . . . . . . . . . . . . . . . . . . . . 7

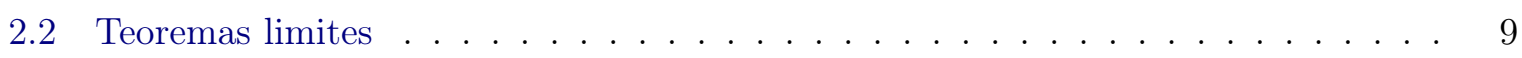

2.3 Provas . . . . . . . . . . . . . . . . . . . . . . 14

2.3 .1 Mudança do tempo . . . . . . . . . . . . . . . . . 15

2.3.2 Redução da dimensão e limite determinístico . . . . . . . . . . . . . . . . 15

2.3.3 Provas dos teoremas . . . . . . . . . . . . . . . . 17

3 Generalização do modelo de Daley-Kendall 19

3.1 O modelo . . . . . . . . . . . . . . . . . . . . . . . . . . . . . . 19

3.2 Teoremas limites . . . . . . . . . . . . . . . . . . . 21

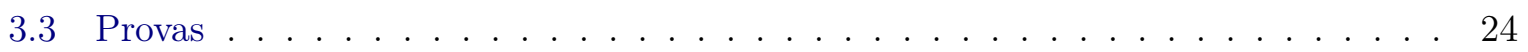

3.3 .1 Mudança do tempo . . . . . . . . . . . . . . . . . . . . 24

3.3 .2 Limite determinístico . . . . . . . . . . . . . . . . . . . . . . 24

3.3 .3 Provas dos teoremas . . . . . . . . . . . . . . . . . 26

4 Uma abordagem via sistemas de partículas $\quad 28$

4.1 O modelo $[\mathrm{ALMM}] \ldots \ldots \ldots \ldots \ldots \ldots$

$4.1 .1 \quad$ O modelo auxiliar . . . . . . . . . . . . . . . . . . . . . . . . 29

4.2 A difusão de um rumor como um sistema de partículas . . . . . . . . . . . . . . 30

4.3 Comentários finais . . . . . . . . . . . . . . . . . . . . . 31

A Construção gráfica dos modelos $\quad 32$

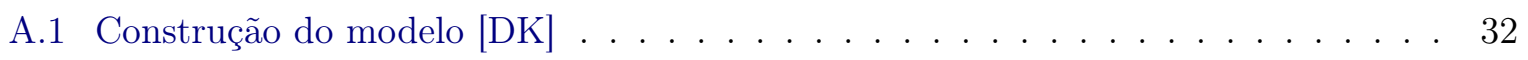

A.2 Construção do modelo $[\mathrm{MT}] \ldots \ldots \ldots$. . . . . . . . . . . . . . . 33 
B Cadeias de Markov "density dependent" $\quad 34$

B.1 Definição e caracterização do processo . . . . . . . . . . . . . . . . 34

B.2 Lei dos Grandes Números . . . . . . . . . . . . . . . . . . . . . 35

B.3 Teorema Central do Limite . . . . . . . . . . . . . . . . . 36

$\begin{array}{ll}\text { Referências Bibliográficas } & 38\end{array}$

$\begin{array}{ll}\text { Índice Remissivo } & 40\end{array}$ 


\section{Lista de Símbolos}

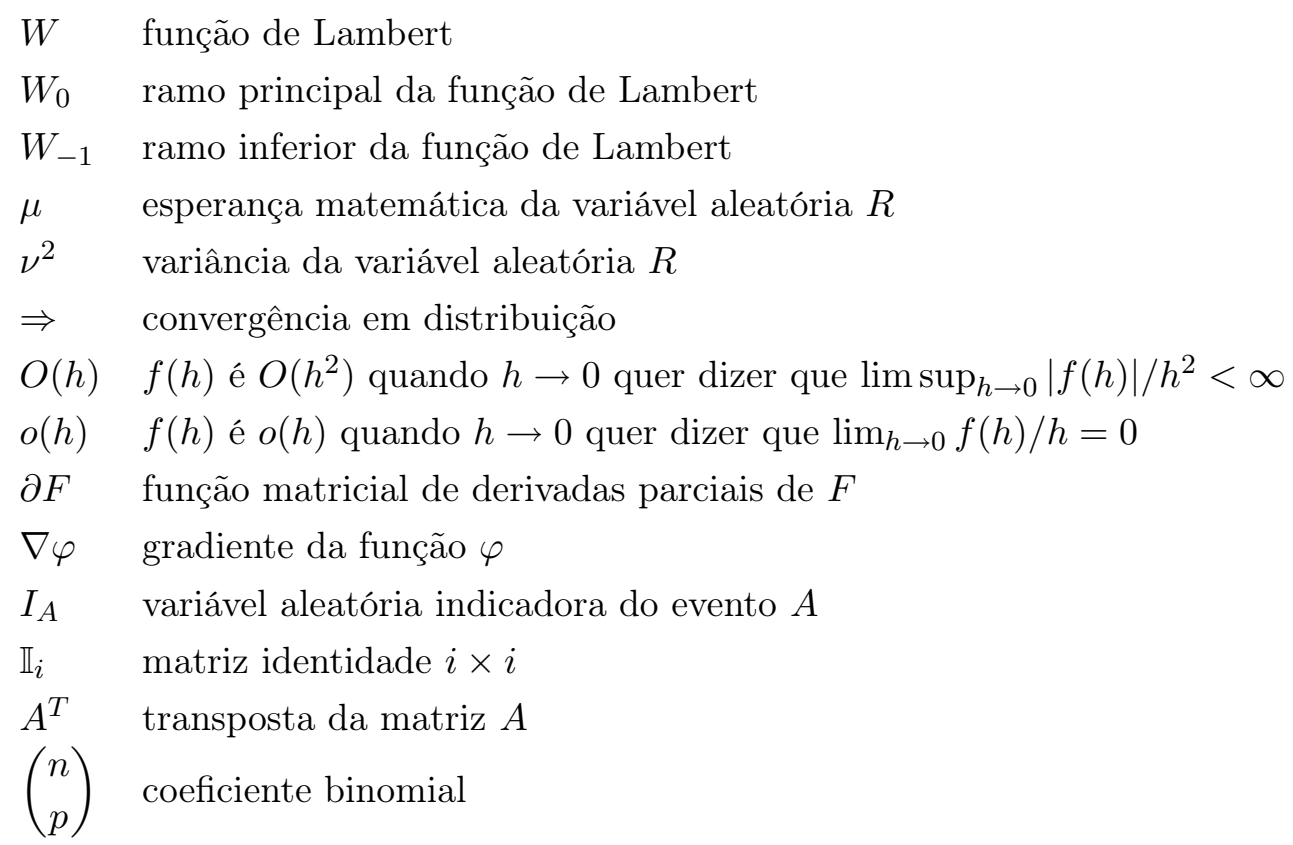




\section{Capítulo 1}

\section{Introdução}

\subsection{Considerações preliminares}

Nas últimas décadas tem havido um grande interesse em entender e modelar diferentes processos de difusão de um rumor em uma população. As primeiras referências matemáticas aparecem em Rapoport e Rebhun (1952) e Rapoport (1953a,b) onde são propostos diversos modelos para a propagação de uma informação usando dados experimentais deste processo. No livro clássico de Feller (1957) (Exercício II.10.21) menciona-se o processo de difusão de um rumor e Goffman e Newill (1964) são os primeiros em observar a analogia entre este processo e a transmissão de uma infecção.

Daley e Kendall (1965) e Maki e Thompson (1973) introduzem os dois modelos estocásticos de rumores mais citados até o momento. Em ambos considera-se uma população finita subdividida em três classes de indivíduos denominados ignorantes, informantes e contidos. Depois de um rumor ser introduzido na população, difunde-se através desta seguindo determinadas regras que dependem da classe à qual a pessoa que sabe do rumor pertence. Tanto a proporção final de indivíduos que nunca chegam a conhecer o rumor quanto o tempo que este demora em ser difundido são variáveis de interesse para os modelos propostos.

A maioria dos modelos introduzidos posteriormente modificam esses modelos básicos no intuito de procurar variantes mais adequadas para descrever os processos da vida real. O interesse nesses processos justifica-se principalmente pela evidente importância do seu comportamento em campos como a economia, a política e as comunicações sociais. Como ilustração de estas aplicações citamos Kosfeld (2005) onde estudam-se efeitos de rumores em mercados financeiros, Schramm (2006) onde disserta-se sobre a difussão de rumores no contexto da propagação de ideologias e Nekovee et al. (2007) onde analisa-se a dinâmica de um rumor em redes sociais complexas.

Um dos objetivos desta tese é introduzir novas generalizações dos modelos clássicos da teoria de rumores estocásticos, isto é, do modelo de Daley-Kendall e do modelo de Maki-Thompson. Para isto, propomos uma nova abordagem que permite trabalhar em um contexto mais geral.

As técnicas encontradas na literatura para estudar modelos de rumores são o princípio de difusão de constantes arbitrárias usado por Daley e Kendall (1965); argumentos de martingais usa- 
dos em Sudbury (1985), Watson (1988), Pittel (1990), Lefevre e Picard (1994) e Carnal (1994); o método de funções geradoras apresentado em Daley e Gani (1999), Gani (2000) e Pearce (2000) e a análise de versões determinísticas como as encontradas em Belen e Pearce (2004) e Kawachi (2008). Neste trabalho apresentamos uma alternativa para essas técnicas baseando-nos na teoria de cadeias de Markov "density dependent" encontrada nos livros de Andersson e Britton (2000) e Ethier e Kurtz (2005). O uso desta nova abordagem nos permite apresentar resultados assintóticos para um modelo geral que tem como casos particulares os famosos modelos de Daley-Kendall e Maki-Thompson, além de variações de modelos de rumores apresentados na literatura recentemente.

Outra contribuição da tese é motivada pela importância dos modelos de sistemas de partículas em grafos como ferramenta para entender a transmissão de uma infeç̧ão em uma população. Para isto, usualmente representam-se indivíduos por vértices e contatos de transmissão por elos entre esses vértices. Desta maneira é obtido um grafo que reune toda a informação sobre a estrutura da população. Os sistemas de partículas são usados em geral para descrever um ou varios processos que tem lugar nessa população. Esta interpretação é bastante usada para modelos epidêmicos mas poucas referências são conhecidas referentes à difusão de uma informação. Diante disso introduzimos esta interpretação para descrever a difusão de um rumor.

A tese é organizada da seguinte maneira. Nas próximas seções deste capítulo resumimos a teoria conhecida dos rumores estocásticos. Na Seção 1.2 fazemos uma breve comparação entre o processo da transmissão de uma infecção e o processo da difusão de um rumor. Na Seção 1.3 introduzimos formalmente o modelo de Daley-Kendall e os principais resultados conhecidos na literatura. Aplicamos o mesmo esquema na Seção 1.4 para o modelo de Maki-Thompson.

No Capítulo 2 introduzimos a generalização do modelo de Maki-Thompson, os principais resultados obtidos e as provas dos teoremas. Analogamente estruturamos o Capítulo 3 referente à generalização do modelo de Daley-Kendall. Finalmente, no Capítulo 4 discutimos como um modelo de sistemas de passeios aleatórios no grafo completo pode descrever um processo da difusão de um rumor. Os conceitos da teoria de cadeias de Markov "density dependent" e teoremas de convergência que são usados nesta tese estão reunidos no Apêndice.

\section{$1.2 \quad$ Epidemias e rumores}

Há semelhanças e diferenças entre o processo de transmissão de uma infecção e o processo de difusão de um rumor. Ambos tem lugar em uma população finita subdividida em diferentes classes de indivíduos e as regras de transmissão ou difusão são similares. Em Dietz (1967) é feita uma revisão destes processos e nos livros de Maki e Thompson (1973) e Daley e Gani (1999) pode-se encontrar uma descrição completa dos principais modelos e resultados existentes da teoria.

O modelo epidêmico SIR é chamado desta forma para ressaltar as classes de indivíduos que são consideradas: susceptíveis, infectados e removidos. O número de indivíduos destas classes em um determinado instante de tempo $t$ é usualmente denotado por $S(t), I(t)$ e $R(t)$ respectivamente 
e é suposto que

$$
S(t)+I(t)+R(t)=N+1
$$

para todo $t \geq 0$.

Neste modelo, definido para uma população homogênea misturada e fechada de $N+1$ indivíduos, o processo $\{(S(t), I(t))\}_{t \geq 0}$ é uma cadeia de Markov a tempo contínuo com transições e correspondentes taxas dadas por

$$
\begin{array}{cc}
\text { transição } & \text { taxa } \\
(-1,1) & S I \\
(0,-1) & I .
\end{array}
$$

Estas transições correspondem-se ao evento em que um infectado transmite a infecção para um susceptível e ao evento em que um infectado é removido da população. Formalmente, a tabela representa as seguintes probabilidades:

$$
\begin{gathered}
P((S(t+h), I(t+h))=(S-1, I+1) \mid(S(t), I(t))=(S, I))=S I h+o(h), \quad \mathrm{e} \\
P((S(t+h), I(t+h))=(S, I-1) \mid(S(t), I(t))=(S, I))=I h+o(h) .
\end{gathered}
$$

No contexto da propagação de um rumor ou uma informação, os análogos dos susceptíveis são os ignorantes, que podemos pensar como estando susceptíveis ao recebimento da nova informação. Os equivalentes aos infectados são os informantes, aqueles que estão transmitindo a informação. Finalmente, os semelhantes aos removidos são os contidos, aqueles que foram informados mas já não produzem influência alguma na população. Nas próximas seções descreveremos melhor estas três classes.

O modelo SIR é um bom exemplo para ilustrar as semelhanças e diferenças entre os modelos epidêmicos e os modelos de rumores. O mecanismo de transmissão é o mesmo em todos os casos. É necessária a interação de um informante/infectado com um ignorante/susceptível para que o segundo passe a fazer parte da classe do primeiro. Por outro lado, os mecanismos para que um informante/infectado saia dessa classe diferem nos modelos. Enquanto no SIR isto acontece isoladamente, isto é, sem a influência de outro indivíduo; nos modelos de rumores isto acontece por interação. Quando um informante encontra-se com outro indivíduo já informado, ele pode pensar que a informação já é conhecida e então perder interesse em transmiti-la.

Apesar destas diferenças, costuma-se adaptar a teoria de modelos epidêmicos para estudar modelos de rumores. Mesmo assim, o número de trabalhos existentes sobre estes últimos é muito menor.

Nos Capítulo 2 e 3 usamos a teoria de cadeias de Markov "density dependent" para estudar generalizações dos modelos clássicos de rumores estocásticos. Até onde sabemos esta é a primeira vez que esta teoria é aplicada neste contexto.

No Capítulo 4 propomos uma outra abordagem por meio de um sistema de partículas no grafo completo para descrever um processo de difusão de um rumor. 


\subsection{O modelo de Daley-Kendall}

Um dos primeiros modelos estocásticos para a difusão de um rumor é introduzido por Daley e Kendall (1965). Neste modelo, chamado modelo [DK] na sequência, considera-se uma população homogênea misturada e fechada de $N+1$ indivíduos. A população é subdividida em três grupos; aqueles que ignoram um determinado rumor, aqueles que conhecem o rumor e o difundem, e aqueles que sabem o rumor mas têm deixado de propagá-lo. Esses grupos são chamados de ignorantes, informantes e contidos, respectivamente. ${ }^{1}$

O rumor é propagado pela população por contatos dois a dois entre seus indivíduos, seguindo as seguintes regras:

1. Os informantes envolvidos em um contato tentam informar ao outro indivíduo do rumor;

2. se o outro indivíduo for um ignorante, ele vira um informante;

3. em qualquer outro caso os informantes envolvidos no encontro assumem que o rumor é conhecido e desistem de transmiti-lo transformando-se em contidos.

4. Interações que não envolvem informantes não fazem efeito no processo.

Para uma descrição formal adotamos a notação usual denotando ao número de ignorantes, informantes e contidos no instante $t$ por $X(t), Y(t)$ e $Z(t)$, respectivamente. Inicialmente,

$$
X(0)=N, Y(0)=1 \text { e } Z(0)=0
$$

e

$$
X(t)+Y(t)+Z(t)=N+1
$$

para todo $t$. O processo $\{(X(t), Y(t))\}_{t \geq 0}$ é uma cadeia de Markov a tempo contínuo com transições e correspondentes taxas dadas por

$$
\begin{array}{cc}
\text { transição } & \text { taxa } \\
(-1,1) & X Y, \\
(0,-2) & \left(\begin{array}{c}
Y \\
2
\end{array}\right), \\
(0,-1) & Y(N+1-X-Y) .
\end{array}
$$

As três transições correspondem às interacções informante-ignorante, informante-informante e informante-contido. Em outras palavras,

$$
\begin{aligned}
& P((X(t+h), Y(t+h))=(X-1, Y+1) \mid(X(t), Y(t))=(X, Y))=X Y h+o(h), \\
& P((X(t+h), Y(t+h))=(X, Y-2) \mid(X(t), Y(t))=(X, Y))=\left(\begin{array}{c}
Y \\
2
\end{array}\right) h+o(h) \quad \mathrm{e}
\end{aligned}
$$

\footnotetext{
${ }^{1} \mathrm{Na}$ literatura de rumores estocásticos esses grupos são conhecidos como ignorants, spreaders e stiflers, respectivamente.
} 


$$
P((X(t+h), Y(t+h))=(X, Y-1) \mid(X(t), Y(t))=(X, Y))=Y(N+1-X-Y) h+o(h),
$$

são as probabilidades de transição representadas pela tabela (1.1). No Apêndice A, apresentamos uma construção gráfica para este modelo.

Uma quantidade de interesse é a proporção de ignorantes no final do processo, isto é, no momento em que não há mais informantes na população. Esta proporção é rigorosamente estudada pela primeira vez para este modelo por Watson (1988), onde demonstra-se que a distribuição deste valor é assintoticamente normal com média $\approx 0.2032$ e variância de $\approx 0.310673 N^{-1}$, quando a proporção inicial de informantes não é desprezível. Por outro lado, Pittel (1990) complementa esses resultados considerando o caso em que esta proporção é pequena.

Daley e Gani (1999) reproduzem duas variantes do modelo [DK] originalmente introduzidas em Daley e Kendall (1965). Na primeira, chamada de variante $k$-fold stifling, assume-se que um informante só decide parar de transimitir o rumor no instante em que encontra-se com o $k$-ésimo indivíduo já informado, seja este informante ou contido. Na segunda, chamada de variante $(\alpha, p)$, assume-se que os informantes tentam difundir o rumor em cada encontro com probabilidade $p$ e que eles transforman-se em contidos com probabilidade $\alpha$ (o modelo [DK] resulta de considerar $\alpha=p=1)$. Em ambos casos, descrevem-se e estudam-se as versões determinísticas.

Notemos que para a variante $(\alpha, p)$ do modelo [DK] a cadeia de Markov a tempo contínuo $\{(X(t), Y(t))\}_{t \geq 0}$ tem às seguintes transições e taxas

$$
\begin{array}{cc}
\text { transição } & \text { taxa } \\
(-1,1) & p X Y, \\
(0,-2) & \alpha^{2} p(2-p)\left(\begin{array}{c}
Y \\
2
\end{array}\right), \\
(0,-1) & \alpha(1-\alpha) p(2-p) Y(Y-1)+\alpha p Y(N+1-X-Y) .
\end{array}
$$

Mais precisamente, temos que

$$
\begin{gathered}
P((X(t+h), Y(t+h))=(X-1, Y+1) \mid(X(t), Y(t))=(X, Y))=p X Y h+o(h), \\
P((X(t+h), Y(t+h))=(X, Y-2) \mid(X(t), Y(t))=(X, Y))=\alpha^{2} p(2-p)\left(\begin{array}{c}
Y \\
2
\end{array}\right) h+o(h), \quad \mathrm{e} \\
P((X(t+h), Y(t+h))=(X, Y-1) \mid(X(t), Y(t))=(X, Y))=(\alpha(1-\alpha) p(2-p) Y(Y-1)) h+ \\
+(\alpha p Y(N+1-X-Y)) h+o(h) .
\end{gathered}
$$

No Capítulo 3 propomos uma generalização da variante $(\alpha, p)$ do modelo [DK], diferenciando um novo grupo de indivíduos que chamamos de neutros. Na realidade, sugerimos considerar por separado o subgrupo dos contidos que nunca tentou transmitir o rumor. Isto é, aqueles indivíduos que sendo inicialmente ignorantes transformam-se em contidos imediatamente após conhecer o rumor. Schramm (2006) chama a atenção da importância deste grupo no contexto da propagação de ideologias. Em nossa generalização da variante $(\alpha, p)$ do modelo [DK] introduzimos um novo parâmetro representando a probabilidade de um ignorante se transformar em neutro no instante 
em que conhece o rumor.

\subsection{O modelo de Maki-Thompson}

Um modelo alternativo para a difusão de um rumor é introduzido em Maki e Thompson (1973) (chamado modelo [MT] na sequência). Neste caso também é considerada uma população homogênea misturada e fechada de $N+1$ indivíduos. A população é subdividida em três grupos como no modelo [DK] e a diferência básica é que o rumor é propagado por contatos dois a dois entre informantes e outros indivíduos da população, sendo que quando um informante tenta difundir o rumor para outro informante, somente o primeiro transforma-se em contido.

Assim, a cadeia de Markov a tempo contínuo $\{(X(t), Y(t))\}_{t \geq 0}$ evolui de acordo às seguintes transições e taxas

$$
\begin{array}{cc}
\text { transição } & \text { taxa } \\
(-1,1) & X Y, \\
(0,-1) & Y(N-X) .
\end{array}
$$

Em outras palavras, temos que

$$
\begin{gathered}
P((X(t+h), Y(t+h))=(X-1, Y+1) \mid(X(t), Y(t))=(X, Y))=X Y h+o(h), \\
P((X(t+h), Y(t+h))=(X, Y-1) \mid(X(t), Y(t))=(X, Y))=Y(N-X) h+o(h)
\end{gathered}
$$

e que as transições correspondem às interacções informante-ignorante e informante-(informante ou contido), respectivamente. No Apêndice A, apresentamos uma construção gráfica para o modelo [MT].

Este modelo, formulado inicialmente como uma cadeia de Markov a tempo discreto, é apresentado como uma alternativa mais simples do modelo [DK]. De fato, o primeiro resultado rigoroso para modelos estocásticos de rumores é obtido por Sudbury (1985) para o modelo [MT]. Usando argumentos de martingais prova-se que a proporção da população que nunca chega conhecer o rumor converge em probabilidade para $\approx 0.203$ quando $N$ tende para $\infty$. Este resultado é generalizado em Watson (1988), onde demonstra-se que a sua distribuição é assintoticamente normal com média $\approx 0.2032$ e variância de $\approx 0.2728 N^{-1}$. Como já mencionamos, no mesmo trabalho é estudado rigorosamente o modelo [DK] pela primeira vez.

Belen e Pearce (2004) apresentam uma análise determinística da proporção de ignorantes quando o processo começa de condições iniciais gerais. Podemos encontrar mais detalhes dessa análise em Belen (2008) onde também apresenta-se a versão $k$-fold stifling para o modelo [MT]. A versão 2-fold stifling é estudada originalmente por Carnal (1994), onde prova-se que a proporção de ignorantes converge em probabilidade para $\approx 0.059$ quando $N$ tende para $\infty$.

No Capítulo 2 propomos uma generalização da versão $k$-fold stifling do modelo [MT]. Em particular, assumimos que um informante só decide parar de transimitir o rumor depois de se encontrar com um número aleatório de indivíduos já informados. Isto é feito considerando condições iniciais gerais como no trabalho de Belen e Pearce (2004). 


\section{Capítulo 2}

\section{Generalização do modelo de Maki-Thompson}

Neste capítulo propomos uma generalização da versão $k$-fold stifling do modelo [MT]. Para isto, assumimos que um informante só decide parar de transimitir o rumor depois de se encontrar com um número aleatório de indíviduos já informados. ${ }^{1}$

\subsection{O modelo}

Consideramos uma população homogênea fechada e totalmente misturada de tamanho $N+1$. Seja $R$ uma variável aleatória inteira não negativa com distribuição dada por

$$
P(R=i)=r_{i} \quad \text { para } i=0,1, \ldots
$$

e sejam

$$
\mu=E[R]>0 \quad \text { e } \quad \nu^{2}=\operatorname{Var}[R] .
$$

Associamos independentemente para cada indivíduo incialmente ignorante uma variável aleatória com a mesma distribuição de $R$. Uma vez que o ignorante sabe do rumor, a copia de $R$ associada a ele determina o número de tentativas frustradas ${ }^{2}$ que o novo informante terá até decidir parar de difundir o rumor. Isto é, o número de encontros deste novo informante com um indivíduo já informado. No caso da variável ser igual a zero, o ignorante se junta aos contidos imediatamente após conhecer o rumor.

Para $i=1,2, \ldots$ dizemos que em um instante determinado de tempo um informante é do tipo $i$ se ele ainda tem $i$ tentativas frustradas antes de parar de transmitir o rumor. Denotamos ao número de ignorantes, informantes de tipo $i$ e contidos no instante $t$ por $X^{(N)}(t), Y_{i}^{(N)}(t)$ e $Z^{(N)}(t)$, respectivamente. Seja

$$
Y^{(N)}(t)=\sum_{i=1}^{\infty} Y_{i}^{(N)}(t)
$$

\footnotetext{
${ }^{1}$ Os resultados deste Capítulo estão reunidos em Lebensztayn et al. (2010b).

${ }^{2}$ A noção de tentativa frustrada aqui considerada é chamada em Daley e Gani (1999) de stifling experience.
} 
o número total de informantes no instante $t$. Então

$$
X^{(N)}(t)+Y^{(N)}(t)+Z^{(N)}(t)=N+1,
$$

para todo $t$. O processo infinito dimensional

$$
\left\{V^{(N)}(t)\right\}_{t \geq 0}:=\left\{\left(X^{(N)}(t), Y_{1}^{(N)}(t), Y_{2}^{(N)}(t), \ldots\right)\right\}_{t \geq 0}
$$

é uma cadeia de Markov a tempo contínuo com transições e correspondentes taxas dadas por

$$
\begin{array}{ccc}
\text { transição } & \text { taxa } & \\
(-1,0, \ldots, 0,0, \ldots) & r_{0} X Y, & \\
(-1,0, \ldots, \stackrel{i-1}{0}, 1, \ldots) & r_{i} X Y, \quad \text { para } i=1,2, \ldots \\
(0,0, \ldots, \stackrel{i-1}{1},-1, \ldots) & (N-X) Y_{i}, \quad \text { para } i=2,3, \ldots \\
(0,-1, \ldots, 0,0, \ldots) & (N-X) Y_{1} . &
\end{array}
$$

Em palavras, o primeiro caso indica a transição do processo na qual um informante interage com um ignorante e o ignorante transforma-se em contido imediatamente (evento que acontece com probabilidade $r_{0}$ ). O segundo caso indica a transição na qual o informante interage com um ignorante e o ignorante transforma-se em um informante do tipo $i$ (evento que acontece com probabilidade $r_{i}$ ). A terceira transição representa a situação na qual um informante de tipo $i$ tem uma tentativa frustrada mas continua sendo um informante (agora de tipo $i-1$ ), e finalmente a última transição indica o evento que um informante de tipo 1 tem uma tentativa frustrada transformando-se portanto em um contido.

Chamamos este modelo de variante random stifling do modelo [MT]. Assumimos que o processo começa com

$$
\begin{aligned}
& X^{(N)}(0)=(N+1) x_{0}^{(N)}, \\
& Y_{i}^{(N)}(0)=(N+1) y_{i, 0}^{(N)}, \quad \text { para } i=1,2, \ldots \mathrm{e} \\
& Z^{(N)}(0)=(N+1) z_{0}^{(N)} .
\end{aligned}
$$

Isto é, $x_{0}^{(N)}, y_{i, 0}^{(N)}, z_{0}^{(N)} \in[0,1]$ são as proporções iniciais de ignorantes, informantes de tipo $i$ e contidos da população, respectivamente, definidas de maneira que

$$
\begin{gathered}
\sum_{i=1}^{\infty} y_{i, 0}^{(N)}>0 \mathrm{e} \\
x_{0}^{(N)}+\sum_{i=1}^{\infty} y_{i, 0}^{(N)}+z_{0}^{(N)}=1 .
\end{gathered}
$$

Além disso, assumimos que os seguintes limites existem: 


$$
\begin{aligned}
x_{0} & =\lim _{N \rightarrow \infty} x_{0}^{(N)}>0 \quad \mathrm{e} \\
y_{i, 0} & =\lim _{N \rightarrow \infty} y_{i, 0}^{(N)} \text { para todo } i=1,2, \ldots
\end{aligned}
$$

e supomos que

$$
w_{0}=\sum_{i=1}^{\infty} i y_{i, 0}<\infty
$$

Como foi mencionado, o processo finaliza eventualmente. Seja

$$
\tau^{(N)}=\inf \left\{t: Y^{(N)}(t)=0\right\}
$$

o tempo de absorção do processo. Nosso principal objetivo é estabelecer teoremas limites para a proporção $N^{-1} X^{(N)}\left(\tau^{(N)}\right)$ de ignorantes ao final do processo.

\subsection{Teoremas limites para a proporção de ignorantes}

Definição 2.2.1. Supomos que $\mu<\infty$ e consideramos a função $f:\left(0, x_{0}\right] \longrightarrow \mathbb{R}$ dada por

$$
f(x)=w_{0}+(1+\mu)\left(x_{0}-x\right)+\log \frac{x}{x_{0}} .
$$

Definimos $x_{\infty}=x_{\infty}\left(\mu, x_{0}, w_{0}\right)$ como sendo a única raiz $x$ de $f$ no intervalo $\left(0, x_{0}\right]$ satisfazendo $f^{\prime}(x) \geq 0$.
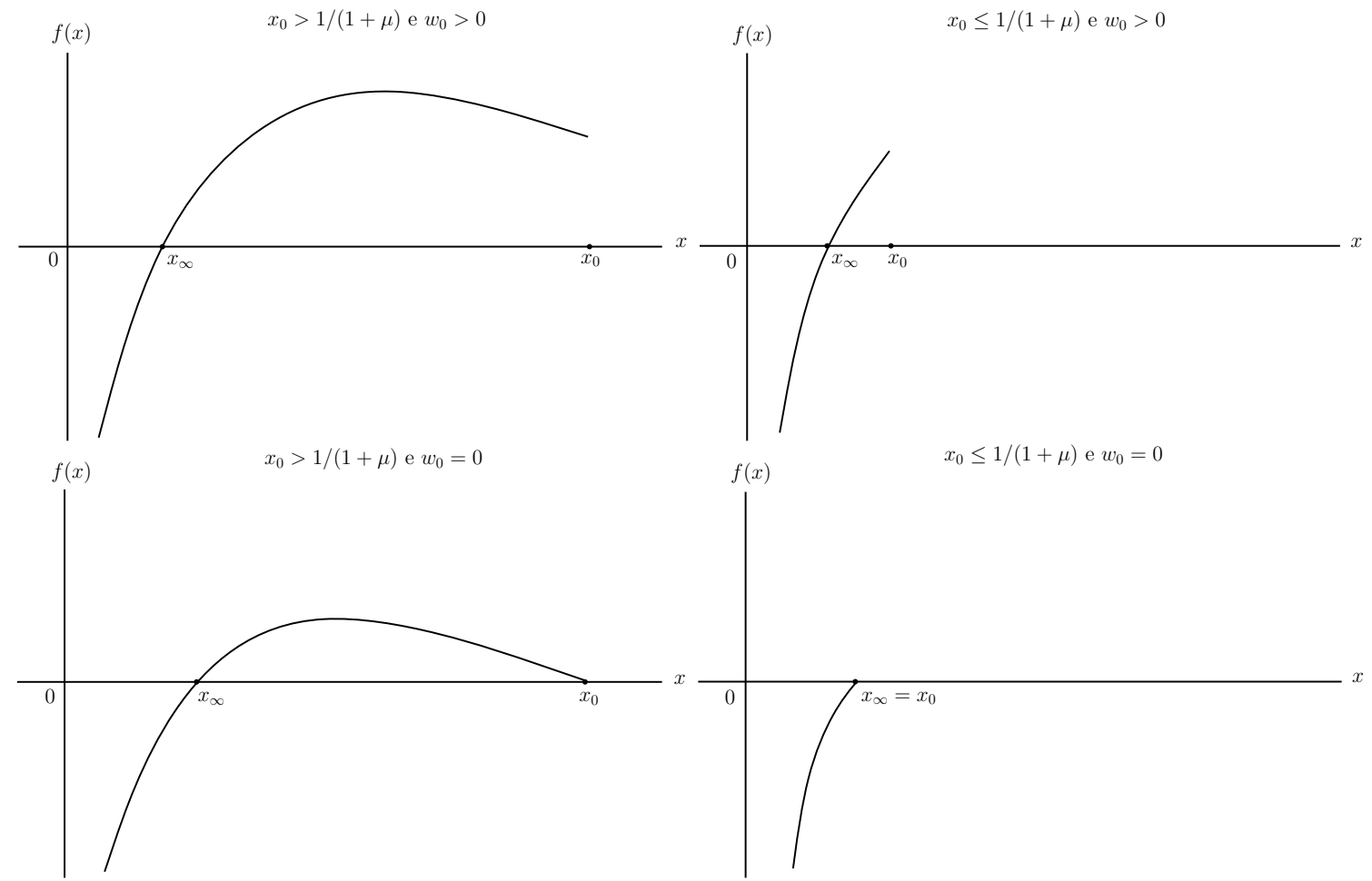

Figura 2.1: Comportamento de $f$ - Os quatro possíveis casos em termos de $x_{0}$ e $w_{0}$. 
Pode-se mostrar que $x_{\infty}$ é a única raiz de $f$, exceto no caso no qual $w_{0}=0$ e $x_{0}>(1+\mu)^{-1}$. Ver Figura 2.1.

Observação 2.2.2. Segue-se da Definição 2.2.1 que $x_{\infty}$ pode-se escrever em termos da função $W$ de Lambert. Esta função é definida como sendo a inversa da função $x \mapsto x e^{x}$, isto é, a função que satisfaz

$$
W(x) e^{W(x)}=x .
$$

Se $-1 / e \leq x<0$ temos dois possíveis valores reais de $W(x)$ (Ver Figura 2.2). Denotamos com $W_{0}(x)$ ao ramo de $W$ tal que $-1 \leq W(x)$ (chamado ramo principal) e com $W_{-1}(x)$ aquele tal que $W(x) \leq-1$ (chamado ramo inferior). Para mais detalhes desta função sugerimos consultar Corless et al. (1996) onde são reunidos interessantes resultados e aplicações.

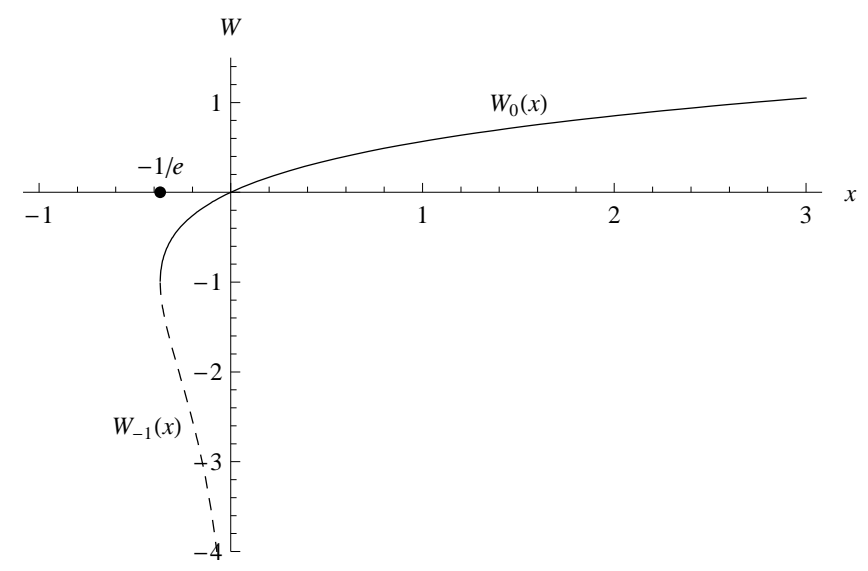

Figura 2.2: Os dois ramos da função $W$ de Lambert.

Notemos que $x_{\infty}$ satisfaz

$$
x_{\infty}=x_{0} e^{-(1+\mu)\left(x_{0}-x_{\infty}\right)-w_{0}}
$$

que podemos escrever como

$$
-x_{0}(1+\mu) e^{-x_{0}(1+\mu)-w_{0}}=-x_{\infty}(1+\mu) e^{-x_{\infty}(1+\mu)} .
$$

Então, obtemos de (2.5) que

$$
x_{\infty}\left(\mu, x_{0}, w_{0}\right)=-(1+\mu)^{-1} W_{0}\left(-x_{0}(1+\mu) e^{-x_{0}(1+\mu)-w_{0}}\right)
$$

observando que $-e^{-1}<-x_{0}(1+\mu) e^{-x_{0}(1+\mu)-w_{0}}<0$.

No que segue estabelecemos uma Lei Fraca dos Grandes Números para a proporção da população que não chega a conhecer o rumor.

Teorema 2.2.3. Se $\mu<\infty$, então

$$
\lim _{N \rightarrow \infty} \frac{X^{(N)}\left(\tau^{(N)}\right)}{N}=x_{\infty} \quad \text { em probabilidade. }
$$




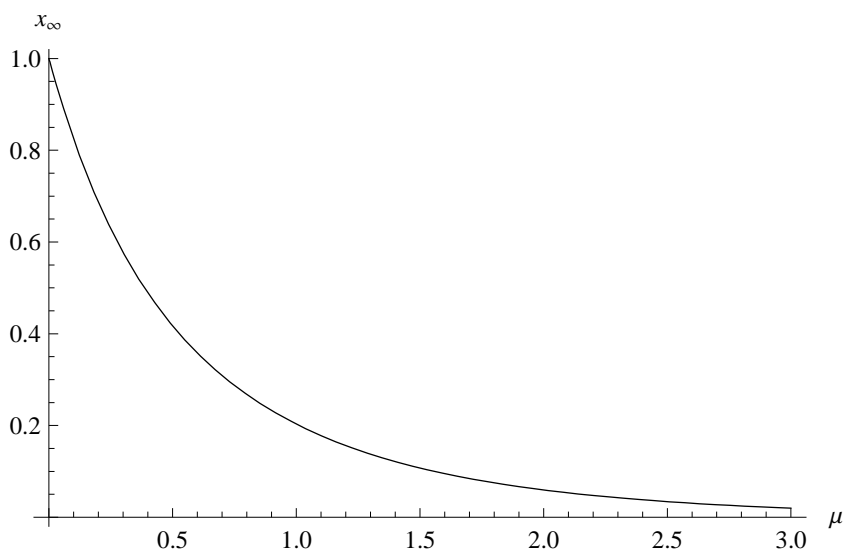

Figura 2.3: Comportamento de $x_{\infty}$ em função de $\mu$ para $x_{0}=1$ e $w_{0}=0$.

Na Figura 2.3 podemos ver o comportamento desta proporção limite em função de $\mu$. Como consequência do teorema anterior conseguimos o seguinte resultado.

Corolário 2.2.4. Se $\mu=\infty$, então

$$
\lim _{N \rightarrow \infty} \frac{X^{(N)}\left(\tau^{(N)}\right)}{N}=0 \quad \text { em probabilidade }
$$

Demonstração do Corolário 2.2.4. Sejam $R_{1}$ e $R_{2}$ variáveis aleatórias tais que $R_{1} \stackrel{\mathcal{D}}{\leq} R_{2}$, isto é, tais que

$$
P\left(R_{1} \geq i\right) \leq P\left(R_{2} \geq i\right)
$$

para todo $i \geq 0$. Consideramos os processos $\left\{V_{1}^{(N)}(t)\right\}_{t \geq 0}$ e $\left\{V_{2}^{(N)}(t)\right\}_{t \geq 0}$ definidos como em (2.1) considerando $R_{1}$ e $R_{2}$, respectivamente; e com as mesmas condições iniciais. Sejam $\tau_{1}^{(N)}$ e $\tau_{2}^{(N)}$ os respectivos tempos de absorção. É possível construir os processos de maneira que

$$
X_{2}^{(N)}\left(\tau_{2}^{(N)}\right) \leq X_{1}^{(N)}\left(\tau_{1}^{(N)}\right), \quad \text { q.c. }
$$

Agora, supomos que $\left\{V^{(N)}(t)\right\}_{t \geq 0}$ é o processo definido antes com a variável aleatória $R$ satisfazendo $\mu=\infty$. Lembrando que

$$
P(R=i)=r_{i} \quad \text { para } i=0,1, \ldots
$$

definimos para cada $k \geq 1$ uma nova variável aletória $R_{k}$ com distribuição dada por

$$
P\left(R_{k}=i\right)=r_{i}, \quad \text { se } i<k \quad \text { e } \quad P\left(R_{k}=k\right)=\sum_{j=k}^{\infty} r_{j} .
$$

Segue desta construção que $R_{k} \stackrel{\mathcal{D}}{\leq} R$ para todo $k$. Notando isto e (2.7) concluimos que

$$
X^{(N)}\left(\tau^{(N)}\right) \leq X_{k}^{(N)}\left(\tau_{k}^{(N)}\right) \text {, q.c. }
$$


para todo $k$. Deste modo (2.8), Teorema 2.2 .3 e (2.6) implicam que

$$
0 \leq \limsup _{N \rightarrow \infty} \frac{X^{(N)}\left(\tau^{(N)}\right)}{N} \leq x_{\infty}\left(\mu_{k}, x_{0}, w_{0}\right) \text { q.c. },
$$

onde $\mu_{k}=E\left[R_{k}\right]$. Como $x_{0}>0$ e $\lim _{k \rightarrow \infty} \mu_{k}=\infty$, temos que $x_{0}>\left(1+\mu_{k}\right)^{-1}$ para $k$ suficientemente grande e neste caso um pequeno cálculo mostra que $x_{\infty}\left(\mu, x_{0}, w_{0}\right)$ converge para 0 quando $k \rightarrow \infty$. Isto completa a prova do Corolario 2.2.4.

Concluimos esta seção apresentando um Teorema Central do Limite para a proporção da população que não chega a conhecer o rumor.

Teorema 2.2.5. Seja $\nu^{2}<\infty$. Se $w_{0}>0$ ou $w_{0}=0$ e $x_{0}>(1+\mu)^{-1}$, então

$$
\sqrt{N}\left(\frac{X^{(N)}\left(\tau^{(N)}\right)}{N}-x_{\infty}\right) \Rightarrow N\left(0, \sigma^{2}\right)
$$

quando $N$ tende para $\infty$, onde $N\left(0, \sigma^{2}\right)$ é a distribuição Gaussiana com média zero e variância dada por

$$
\sigma^{2}=\sigma^{2}\left(\mu, \nu^{2}, x_{0}, w_{0}\right)=\frac{x_{\infty}\left(1-\left(x_{0}^{-1}+w_{0}+\left(x_{0}-x_{\infty}\right)\left(1+\mu-\nu^{2}\right)\right) x_{\infty}\right)}{\left(1-(1+\mu) x_{\infty}\right)^{2}} .
$$

Observação 2.2.6. Quando $w_{0}=0$ e $x_{0} \leq(1+\mu)^{-1}$ temos que a proporção assintótica de ignorantes é igual à proporção do instante inicial e portanto não há um teorema central do limite (ver Figura 2.4 e Observação 2.3.2). Notemos que isto e a condição do teorema quando $w_{0}=0$ recordam às condições para a extinção ou não de um processo de ramificação. De fato, nosso problema pode ser comparado com o problema de sobrevivência da população de informantes no processo limite. Neste contexto, consideramos a descendência de um informante como sendo todos aqueles indivíduos inicialmente ignorantes que foram informados por ele do rumor. Assim, como no limite a "probabilidade" de un indivíduo ser ignorante é $x_{0}$ e como cada informante tenta contar o rumor até se encontrar com $R$ indivíduos já informados, concluimos que a média da distribuição do número de descendentes é

$$
\mu \frac{x_{0}}{1-x_{0}}
$$

que é maior do que 1 se, e somente se, $(\mu+1) x_{0}>1$. Com efeito, podemos escrever ao número de descendentes de um informante como

$$
\sum_{i=1}^{R} D_{j}
$$

onde $D_{j}$ é o número de ignorantes que um informante encontra até se encontrar com o primeiro indivíduo já informado. 

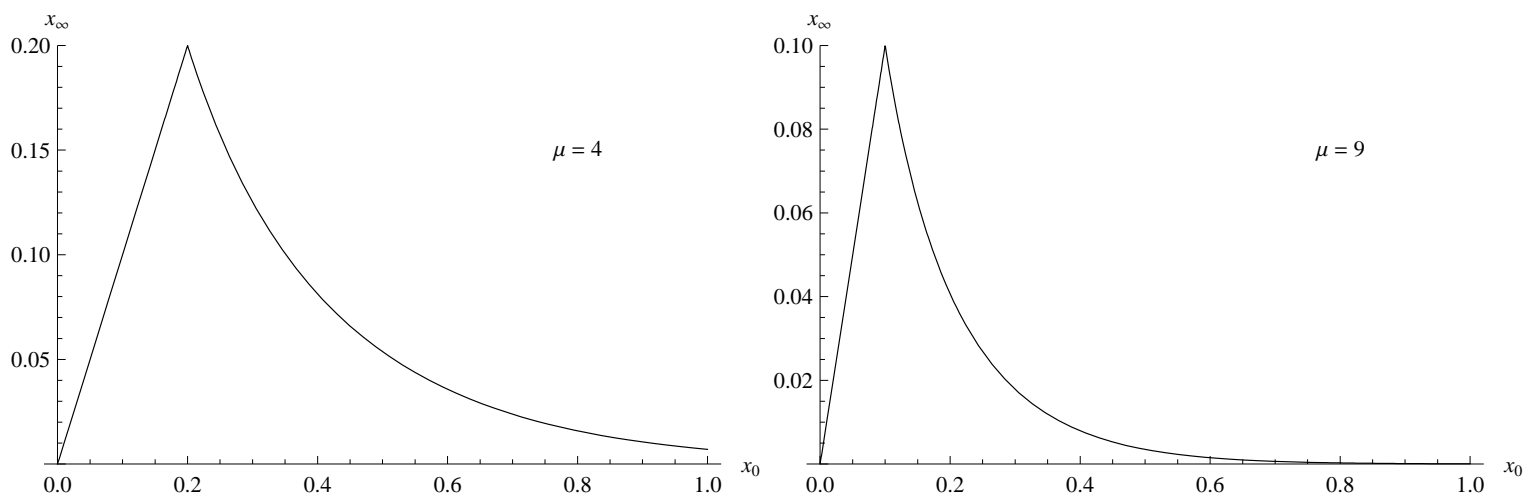

Figura 2.4: Comportamento de $x_{\infty}$ em função de $x_{0}$ para $w_{0}=0$ e diferentes valores de $\mu$.

Observação 2.2.7. Nossos resultados referem-se a condições iniciais gerais, análogo ao considerado na análise determinística de Belen e Pearce (2004). O processo começando com um informante e $N$ ignorantes corresponde a $x_{0}=1$ e $w_{0}=0$, em cujo caso a assintota da variância reduze-se a

$$
\sigma^{2}=\frac{x_{\infty}\left(1-x_{\infty}\right)\left(1-\left(1+\mu-\nu^{2}\right) x_{\infty}\right)}{\left(1-(1+\mu) x_{\infty}\right)^{2}}
$$

Em particular, para $R \equiv k$ ( $k \geq 1$ um inteiro) temos a versão $k$-fold stifling do modelo [MT] apresentada em Belen (2008) e para a qual

$$
\sigma^{2}=\frac{x_{\infty}\left(1-x_{\infty}\right)}{1-(k+1) x_{\infty}}
$$

onde

$$
x_{\infty}=x_{\infty}(k, 1,0)=-(k+1)^{-1} W_{0}\left(-(k+1) e^{-(k+1)}\right) .
$$

Observamos que, neste caso

$$
\lim _{k \rightarrow \infty} \sigma^{2} / x_{\infty}=1
$$

como consequência de que

$$
\lim _{k \rightarrow \infty} W_{0}\left(-(k+1) e^{-(k+1)}\right)=W_{0}(0)=0 .
$$

A Tabela 2.1 exibe os valores de $x_{\infty}$ e $\sigma^{2}$ neste modelo para $k=1, \ldots, 6$. Notemos que $k=2$ é o caso estudado por Carnal (1994) e que a partir de $k=5$ fica em evidência a relação obtida em (2.11).

\begin{tabular}{|c|cccccc|}
\hline$k$ & 1 & 2 & 3 & 4 & 5 & 6 \\
\hline$x_{\infty}$ & 0.203 & 0.0595 & 0.0198 & 0.00698 & 0.00252 & 0.000918 \\
$\sigma^{2}$ & 0.273 & 0.0681 & 0.0211 & 0.00718 & 0.00255 & 0.000923 \\
\hline
\end{tabular}

Tabela 2.1: Versão $k$-fold stifling do modelo [MT], $x_{0}=1$ e $w_{0}=0$. 
O modelo $[\mathrm{MT}]$ original é obtido considerando $R \equiv 1, x_{0}=1$ e $w_{0}=0$. Como consequência, nossos teoremas generalizam os resultados clássicos apresentados em Sudbury (1985) e Watson (1988).

No intuito de reforçar ainda mais a generalidade do modelo que propomos mostramos alguns exemplos não considerados na literatura.

Exemplo 2.2.8. Uma variante interessante é obtida quando $R$ tem uma distribuição geométrica com parâmetro $p$. Isto é, $r_{0}=0$ e $r_{i}=p(1-p)^{i-1}$ para $i=1,2, \ldots$ Neste modelo, um ignorante sempre transforma-se em informante depois de conhecer o rumor e cada vez que um informante encontra-se com alguém já informado, ele decide com probabilidade $p$ se transformar em contido, independentemente para cada informante e cada encontro. A Tabela 2.2 mostra os valores de $x_{\infty}$ e $\sigma^{2}$ para $x_{0}=1, w_{0}=0$ e alguns valores de $p$ arbitrariamente escolhidos.

\begin{tabular}{|c|ccccccc|}
\hline$p$ & 0.3 & 0.4 & 0.5 & 0.6 & 0.7 & 0.8 & 0.9 \\
\hline$x_{\infty}$ & 0.0139 & 0.0340 & 0.0595 & 0.0878 & 0.117 & 0.147 & 0.175 \\
$\sigma^{2}$ & 0.0163 & 0.0427 & 0.0780 & 0.118 & 0.159 & 0.199 & 0.238 \\
\hline
\end{tabular}

Tabela 2.2: $R \sim \operatorname{Geometrica}(p), x_{0}=1$ e $w_{0}=0$.

Exemplo 2.2.9. Como outra variante consideramos $R$ com distribuição Poisson de parâmetro $\lambda$, em cujo caso um ignorante escolhe (com probabilidade $e^{-\lambda}$ ) se transformar em contido imediatamente após saber do rumor. Notemos que no caso em que o ignorante decide transformarse em informante, sua decisão de parar de transmitir o rumor em cada encontro dependerá da história do processo. A tabela 3.1 mostra os valores de $x_{\infty}$ e $\sigma^{2}$ para $x_{0}=1, w_{0}=0$ e alguns valores de $\lambda$ arbitrariamente escolhidos.

\begin{tabular}{|c|cccccccccc|}
\hline$\lambda$ & 0.1 & 0.3 & 0.5 & 0.7 & 0.9 & 1.1 & 1.3 & 1.5 & 1.7 & 1.9 \\
\hline$x_{\infty}$ & 0.824 & 0.577 & 0.417 & 0.309 & 0.233 & 0.178 & 0.138 & 0.107 & 0.0844 & 0.0668 \\
$\sigma^{2}$ & 2.908 & 1.654 & 1.012 & 0.654 & 0.440 & 0.307 & 0.219 & 0.160 & 0.119 & 0.0895 \\
\hline
\end{tabular}

Tabela 2.3: $R \sim \operatorname{Poisson}(\lambda), x_{0}=1$ e $w_{0}=0$.

Neste exemplo mostramos um tipo interessante de modelos que podem ser considerados como caso particular da nossa generalização. Mais precisamente, podemos considerar modelos de rumores nos quais os informantes têm memória de todo o processo. Até agora, os únicos modelos considerados com estas características são as versões $k$-fold stifling dos modelos [MT] e [DK].

\subsection{Provas}

Enunciamos as principais idéias para provar os Teoremas 2.2.3 e 2.2.5. Primeiro, de acordo a uma conveniente mudança do tempo do processo, definimos um novo processo $\left\{\tilde{V}^{(N)}(t)\right\}_{t \geq 0}$ com as mesmas transições que $\left\{V^{(N)}(t)\right\}_{t \geq 0}$ de maneira que ambos finalizam no mesmo ponto 
do espaço de estados. Logo, trabalhamos com uma cadeia de Markov reduzida obtida a partir de $\left\{\tilde{V}^{(N)}(t)\right\}_{t \geq 0}$ para poder aplicar a teoria de cadeias de Markov "density dependent" apresentada em Ethier e Kurtz (2005) e resumida no Apêndice B.

\subsubsection{Mudança do tempo}

Como a distribuição de $X^{(N)}\left(\tau^{(N)}\right)$ depende do processo $\left\{V^{(N)}(t)\right\}_{t \geq 0}$ somente através da cadeia de Markov imersa, consideramos uma versão a tempo mudado do processo. Seja $\left\{\tilde{V}^{(N)}(t)\right\}_{t \geq 0}$ a cadeia de Markov infinito-dimensional e a tempo contínuo

$$
\left\{\left(\tilde{X}^{(N)}(t), \tilde{Y}_{1}^{(N)}(t), \tilde{Y}_{2}^{(N)}(t), \ldots\right)\right\}_{t \geq 0}
$$

com transições e correspondentes taxas dadas por

$$
\begin{array}{ccc}
\operatorname{transição~} & \operatorname{taxa} \\
(-1,0, \ldots, 0,0, \ldots) & r_{0} \tilde{X}, & \\
(-1,0, \ldots, \stackrel{i-1}{0}, 1, \ldots) & r_{i} \tilde{X}, & \text { para } i=1,2, \ldots \\
\left(0,0, \ldots, i_{1}^{1},-1, \ldots\right) & (N-\tilde{X}) \tilde{Y}_{i}(\tilde{Y})^{-1}, & \text { para } i=2,3, \ldots \\
(0,-1, \ldots, 0,0, \ldots) & (N-\tilde{X}) \tilde{Y}_{1}(\tilde{Y})^{-1} . &
\end{array}
$$

Observemos que podemos definir $\left\{\tilde{V}^{(N)}(t)\right\}_{t>0}$ de tal forma que tem o mesmo estado inicial e as mesmas transições de $\left\{V^{(N)}(t)\right\}_{t \geq 0}$. Assim ambos processos têm a mesma cadeia de Markov imersa e portanto, se definimos

$$
\tilde{\tau}^{(N)}=\inf \left\{t: \tilde{Y}^{(N)}(t)=0\right\}
$$

concluímos que $X^{(N)}\left(\tau^{(N)}\right)=\tilde{X}^{(N)}\left(\tilde{\tau}^{(N)}\right) \cdot{ }^{3}$

\subsubsection{Redução da dimensão e limite determinístico}

Para provar os teoremas usando diretamente o Teorema B.2.1, trabalhamos com uma cadeia de Markov reduzida. Definimos

$$
\tilde{W}^{(N)}(t)=\sum_{i=1}^{\infty} i \tilde{Y}_{i}^{(N)}(t),
$$

\footnotetext{
${ }^{3}$ Formalizamos este tipo de transformação para a generalização do modelo DK, no Capítulo 3.
} 
e notamos que o processo $\left\{\left(\tilde{X}^{(N)}(t), \tilde{W}^{(N)}(t)\right)\right\}_{t \geq 0}$ é uma cadeia de Markov a tempo contínuo com transições e taxas dadas por

$$
\begin{array}{cl}
\text { transição } & \text { taxa } \\
\ell_{i}=(-1, i) & r_{i} \tilde{X}, \quad i=0,1, \ldots \\
\ell_{-1}=(0,-1) & N-\tilde{X} .
\end{array}
$$

Agora, definimos para $t \geq 0$

$$
\tilde{v}^{(N)}(t)=\left(\tilde{x}^{(N)}(t), \tilde{w}^{(N)}(t)\right)=N^{-1}\left(\tilde{X}^{(N)}(t), \tilde{W}^{(N)}(t)\right)
$$

e consideramos as funções

$$
\beta_{\ell_{-1}}(x, w)=1-x \text { e } \beta_{\ell_{i}}(x, w)=r_{i} x, \text { para } i=0,1, \ldots
$$

Assim, observamos que as taxas em (2.12) podem-se escrever como

$$
N\left[\beta_{\ell_{i}}\left(\frac{\tilde{X}}{N}, \frac{\tilde{W}}{N}\right)\right]
$$

e então $\left\{\tilde{v}^{(N)}(t)\right\}_{t \geq 0}$ é uma cadeia de Markov "density dependent" com possíveis transições no conjunto $(1 / N)\left\{\ell_{-1}, \ell_{0}, \ell_{1}, \ldots\right\}$.

Diante disso, podemos usar o Teorema B.2.1 para concluir que o processo $\left\{\tilde{v}^{(N)}(t)\right\}_{t \geq 0}$ converge quase certamente quando $N \rightarrow \infty$ para um limite determinístico. A função drift definida em (B.3) neste caso é dada por

$$
F(x, w)=\sum_{i=-1}^{\infty} \ell_{i} \beta_{\ell_{i}}(x, w)=(-x,(\mu+1) x-1) .
$$

Portanto, o sistema determinístico limite é governado pelo sistema de equações diferenciais ordinárias

$$
\left\{\begin{array}{l}
x^{\prime}(t)=-x(t) \\
w^{\prime}(t)=(\mu+1) x-1
\end{array}\right.
$$

com condições iniciais $x(0)=x_{0}$ e $w(0)=w_{0}$. A solução deste sistema é dada por

$$
v(t)=(x(t), w(t)),
$$

onde

$$
x(t)=x_{0} e^{-t}
$$

e

$$
w(t)=f(x(t))=w_{0}+(1+\mu)\left(x_{0}-x(t)\right)-t .
$$


De acordo ao Teorema B.2.1, temos (em um espaço de probabilidade conveniente) que

$$
\lim _{N \rightarrow \infty} \tilde{v}^{(N)}(t)=v(t) \quad \text { q. c. }
$$

uniformemente em intervalos de tempo limitados. Em particular, podemos provar o seguinte lema.

Lema 2.3.1. Vale que

$$
\lim _{N \rightarrow \infty} \tilde{x}^{(N)}(t)=x(t) \quad q . c .
$$

uniformemente em $\mathbb{R}$.

Demonstração. Dado $\varepsilon>0$, tomamos $t_{0}=t_{0}(\varepsilon)$ tal que $x(t) \leq \varepsilon / 2$ para todo $t \geq t_{0}$. Pela convergência uniforme no intervalo $\left[0, t_{0}\right]$, existe $N_{0}=N_{0}(\varepsilon)$ tal que

$$
\left|\tilde{x}^{(N)}(t)-x(t)\right| \leq \varepsilon / 2 \text { para todo } N \geq N_{0} \text { e } t \in\left[0, t_{0}\right] .
$$

Portanto, para todo $N \geq N_{0}$ e $t \geq t_{0}$,

$$
\tilde{x}^{(N)}(t) \leq \tilde{x}^{(N)}\left(t_{0}\right) \leq x\left(t_{0}\right)+\varepsilon / 2 \leq \varepsilon
$$

de onde $\left|\tilde{x}^{(N)}(t)-x(t)\right| \leq \varepsilon$.

\subsubsection{Provas dos teoremas}

Para provar ambos teoremas usamos o Teorema B.3.2. Adoptamos a notação apresentada no apêndice, sendo neste caso $\mathcal{V}=\left(\mathcal{V}_{x}, \mathcal{V}_{w}\right), \varphi(x, w)=w \mathrm{e}$

$$
\tau_{\infty}=\inf \{t: w(t) \leq 0\}=w_{0}+(1+\mu)\left(x_{0}-x_{\infty}\right)
$$

Logo,

$$
\nabla \varphi\left(v\left(\tau_{\infty}\right)\right) \cdot F\left(v\left(\tau_{\infty}\right)\right)=w^{\prime}\left(\tau_{\infty}\right)=(\mu+1) x_{\infty}-1<0 .
$$

Demonstração do Teorema 2.2.3. Notemos que $w_{0}>0$ e (2.14) implicam que $w\left(\tau_{\infty}-\varepsilon\right)>0$ e $w\left(\tau_{\infty}+\varepsilon\right)<0$ para $0<\varepsilon<\tau_{\infty}$. Então, a convergência quase certa de $\tilde{w}^{(N)}$ para $w$ uniformemente em intervalos limitados implica que

$$
\lim _{N \rightarrow \infty} \tilde{\tau}^{(N)}=\tau_{\infty} \quad \text { q.c. }
$$

No caso em que $w_{0}=0$ e $x_{0}>(1+\mu)^{-1}$, o resultado ainda é valido pois $w^{\prime}(0)>0$ e $(2.14)$ segue se verificando. Portanto, como $X^{(N)}\left(\tau^{(N)}\right)=\tilde{X}^{(N)}\left(\tilde{\tau}^{(N)}\right)$, obtemos Teorema 2.2.3 de (2.13) e (2.15).

Observação 2.3.2. Notemos que no caso não considerado no enunciado do Teorema $2.2 .3, w_{0}=0$ e $x_{0} \leq(1+\mu)^{-1}$, temos que $w(t)<0$ para todo $t>0$, e novamente a convergência quase certa de $\tilde{w}^{(N)}$ para $w$ uniformemente em intervalos limitados implica que $\lim _{N \rightarrow \infty} \tilde{\tau}^{(N)}=0=\tau_{\infty}$ quase certamente. 
Demonstração do Teorema 2.2.5. Do Teorema B.3.2, temos que $\sqrt{N}\left(\tilde{x}^{(N)}\left(\tilde{\tau}^{(N)}\right)-x_{\infty}\right)$ converge em distribuição quando $N \rightarrow \infty$ para

$$
\mathcal{V}_{x}\left(\tau_{\infty}\right)+\frac{x_{\infty}}{(\mu+1) x_{\infty}-1} \mathcal{V}_{w}\left(\tau_{\infty}\right)
$$

A distribuição normal resultante tem média zero. Então, para completar a prova do Teorema 2.2.5 falta calcular a correspondente variância.

Para isto, temos que calcular a matriz de covariância $\operatorname{Cov}\left(\mathcal{V}\left(\tau_{\infty}\right), \mathcal{V}\left(\tau_{\infty}\right)\right)$. O primeiro passo é calcular a matriz de derivadas parciais da função drift $F$ e a matriz $G$. Obtemos

$$
\partial F(x, w)=\left(\begin{array}{cc}
-1 & 0 \\
(\mu+1) & 0
\end{array}\right)
$$

$\mathrm{e}$

$$
G(x, w)=\left(\begin{array}{cc}
x & -\mu x \\
-\mu x & \left(\nu^{2}+\mu^{2}-1\right) x+1
\end{array}\right) .
$$

Logo, encontramos a solução $\Phi$ da equação matricial

$$
\frac{\partial}{\partial t} \Phi(t, s)=\partial F(x(t), w(t)) \Phi(t, s), \quad \Phi(s, s)=\mathbb{I}_{2},
$$

a qual é dada por

$$
\Phi(t, s)=\left(\begin{array}{cc}
e^{-(t-s)} & 0 \\
(\mu+1)\left(1-e^{-(t-s)}\right) & 1
\end{array}\right) .
$$

Portanto, a matriz de covariâncias do processo Gaussiano $\mathcal{V}$ no instante $t$ é obtido pela fórmula

$$
\operatorname{Cov}(\mathcal{V}(t), \mathcal{V}(t))=\int_{0}^{t} \Phi(t, s) G(x(s), w(s))[\Phi(t, s)]^{T} d s
$$

Como passo final para calcular $\operatorname{Cov}\left(\mathcal{V}\left(\tau_{\infty}\right), \mathcal{V}\left(\tau_{\infty}\right)\right)$, temos que substituir $e^{-t}$ e $t$ na fórmula obtida de (2.17) por $x_{\infty} / x_{0}$ e $\tau_{\infty}$, respectivamente. As fórmulas resultantes são

$$
\begin{aligned}
\operatorname{Var}\left(\mathcal{V}_{x}\left(\tau_{\infty}\right)\right)= & \left(\left(x_{0}-x_{\infty}\right) x_{\infty}\right) / x_{0} \\
\operatorname{Var}\left(\mathcal{V}_{w}\left(\tau_{\infty}\right)\right)= & (\mu+1)^{2}\left(x_{0}-x_{\infty}\right) x_{\infty} / x_{0}+\nu^{2}\left(x_{0}-x_{\infty}\right) \\
& +\left(1-2(\mu+1) x_{\infty}\right) \tau_{\infty} \\
\operatorname{Cov}\left(\mathcal{V}_{x}\left(\tau_{\infty}\right), \mathcal{V}_{w}\left(\tau_{\infty}\right)\right)= & \tau_{\infty} x_{\infty}-(\mu+1)\left(x_{0}-x_{\infty}\right) x_{\infty} / x_{0}
\end{aligned}
$$

Usando que $\tau_{\infty}=w_{0}+(1+\mu)\left(x_{0}-x_{\infty}\right),(2.16)$ e propriedades da variância, obtemos a fórmula (2.10). 


\section{Capítulo 3}

\section{Generalização do modelo de Daley-Kendall}

Neste capítulo propomos uma generalização da variante $(\alpha, p)$ do modelo [DK], diferenciando um novo grupo de indíviduos que chamamos de neutros. Introduzimos um novo parâmetro representando a probabilidade de um ignorante se transformar em neutro no instante em que conhece o rumor. ${ }^{1}$

\section{$3.1 \quad$ O modelo}

Consideramos $\alpha, p, q \in(0,1]$. Suponha que um informante envolvido em um encontro decide contar o rumor com probabilidade $p$ e que, uma vez que a decisão é tomada, qualquer informante em um encontro com alguém já informado tem probabilidade $\alpha$ de transformar-se em contido, independentemente para cada informante e cada encontro. Além disto, assumimos que quando um ignorante escuta o rumor de um informante, ele escolhe transformar-se em informante ou em neutro (isto é, imediatamente em um contido) com respectivas probabilidades $q$ e $1-q$. Denotamos por $U(t)$ o número de indivíduos neutros no instante $t$, e seja $V(t)=(X(t), U(t), Y(t))$. Então, $\{V(t)\}_{t \geq 0}$ é uma cadeia de Markov a tempo contínuo começando em $(N, 0,1)$ com transições e correspondentes taxas dadas por

$$
\begin{array}{cc}
\text { transição } & \text { taxa } \\
(-1,0,1) & p q X Y, \\
(-1,1,0) & p(1-q) X Y, \\
(0,0,-2) & \alpha^{2} p(2-p)\left(\begin{array}{c}
Y \\
2
\end{array}\right), \\
(0,0,-1) & \alpha(1-\alpha) p(2-p) Y(Y-1)+\alpha p Y(N+1-X-Y) .
\end{array}
$$

Chamamos este modelo de variante $(\alpha, p, q)$ do modelo [DK]. O caso $q=1$ corresponde à variante $(\alpha, p)$ definida em Daley e Gani (1999) e o modelo [DK] básico é obtido tomando $\alpha=p=q=1$.

\footnotetext{
${ }^{1}$ Os resultados deste Capítulo estão reunidos em Lebensztayn et al. (2010a).
} 
Uma abordagem análoga à considerada no Capítulo 2 pode nos conduzir a teoremas limites para esta generalização. De fato, vamos obter resultados rigorosos para uma família de modelos de rumores que evidentemente inclui o modelo aqui proposto.

Consideramos o seguinte modelo mais geral. Novamente, seja $V=(X, U, Y)$ e suponha que $\{V(t)\}_{t \geq 0}$ é uma cadeia de Markov a tempo contínuo com estado inicial $(N, 0,1)$ e transições e taxas dadas por

$$
\begin{array}{cc}
\text { transição } & \operatorname{taxa} \\
(-1,0,1) & \lambda \delta X Y, \\
(-1,1,0) & \lambda(1-\delta) X Y, \\
(0,0,-2) & \lambda \theta_{1}\left(\begin{array}{c}
Y \\
2
\end{array}\right), \\
(0,0,-1) & \lambda \theta_{2} Y(Y-1)+\lambda \gamma Y(N+1-X-Y) .
\end{array}
$$

Definimos $\theta=\theta_{1}+\theta_{2}-\gamma$ e assumimos que

$$
\lambda>0, \gamma>0, \theta_{1} \geq 0, \theta_{2} \geq 0,0<\delta \leq 1 \text { e } 0 \leq \theta \leq 1 .
$$

Com isto, temos uma cadeia de Markov que dependendo dos valores dos parâmetros inclui modelos de rumores com diferentes mecanismos de difusão. Isto fica em evidência na Tabela 3.1, que resume alguns dos modelos que resultam como caso particular desta cadeia geral. Daremos mais detalhes destes modelos na próxima seção.

\begin{tabular}{|c|c|c|c|c|c|}
\hline$\lambda$ & $\delta$ & $\gamma$ & $\theta_{1}$ & $\theta_{2}$ & Modelo \\
\hline 1 & 1 & 1 & 1 & 0 & Daley e Kendall (1965) \\
1 & 1 & 1 & 0 & 1 & Maki e Thompson (1973) \\
$p$ & 1 & $\alpha$ & $\alpha^{2}(2-p)$ & $\alpha(1-\alpha)(2-p)$ & Daley e Gani (1999) \\
$p$ & 1 & $r / p$ & $q_{2} / p$ & $q_{1} /(2 p)$ & Pearce (2000) \\
1 & 1 & 1 & 2 & 0 & Hayes (2005) \\
$\tilde{\alpha}$ & $\tilde{\theta}$ & $\tilde{\gamma} / \tilde{\alpha}$ & $\tilde{\beta} / \tilde{\alpha}$ & 0 & Kawachi (2008) \\
$p$ & $q$ & $\alpha$ & $\alpha^{2}(2-p)$ & $\alpha(1-\alpha)(2-p)$ & Lebensztayn et al. (2010) \\
\hline
\end{tabular}

Tabela 3.1: Modelos de rumores existentes na literatura.

Em particular, a variante $(\alpha, p, q)$ do modelo [DK] é obtida escolhendo

$$
\lambda=p, \delta=q, \theta_{1}=\alpha^{2}(2-p), \theta_{2}=\alpha(1-\alpha)(2-p), \gamma=\alpha \text { e } \theta=\alpha(1-p) .
$$

Definimos

$$
\tau^{(N)}=\inf \left\{t: Y^{(N)}(t)=0\right\}
$$

Na seguinte seção, apresentamos uma Lei Fraca dos Grandes Números e um Teorema Central do Limite para 


$$
N^{-1}\left(X^{(N)}\left(\tau^{(N)}\right), U^{(N)}\left(\tau^{(N)}\right)\right)
$$

\subsection{Teoremas limites para as proporções de ignorantes e neutros}

Começamos com algumas definições.

Definição 3.2.1. Para $0<\theta<1$, consideramos a função $f:[0,1] \rightarrow \mathbb{R}$ dada por

$$
f(x)=\frac{(\gamma+\delta \theta) x^{\theta}-(\gamma+\delta) \theta x-\gamma(1-\theta)}{\theta(1-\theta)} .
$$

Definimos $x_{\infty}=x_{\infty}(\delta, \gamma, \theta)$ como a única raiz de $f$ no intervalo $(0,1)$.

Para justificar a existência e unicidade da raiz, notemos que $f(0)<0, f(1)=0$ e que $f$ é unimodal, com um máximo global no ponto

$$
\left(\frac{\gamma+\delta \theta}{\gamma+\delta}\right)^{\frac{1}{1-\theta}} \in(0,1)
$$

Nos casos $\theta=0$ e $\theta=1, x_{\infty}$ é definido analogamente.

Definição 3.2.2. Consideremos as funções $f_{0}$ e $f_{1}$ definidas em $(0,1]$ por

$$
\begin{aligned}
& f_{0}(x)=\lim _{\theta \rightarrow 0^{+}} f(x)=(\gamma+\delta)(1-x)+\gamma \log x, \\
& f_{1}(x)=\lim _{\theta \rightarrow 1^{-}} f(x)=-\gamma(1-x)-(\gamma+\delta) x \log x .
\end{aligned}
$$

Para cada $\theta \in\{0,1\}$, denotamos por $x_{\infty}(\delta, \gamma, \theta)$ a única raiz de $f_{\theta}$ em $(0,1)$.

Observação 3.2.3. No último caso, podemos escrever $x_{\infty}$ em termos da função $W$ de Lambert. Lembremos que $W_{0}$ e $W_{-1}$ denotam o ramo principal e o ramo inferior desta função, respectivamente (ver Observação 2.2.2). Definindo $h=1+\delta / \gamma$, temos que

$$
\begin{aligned}
& x_{\infty}(\delta, \gamma, 0)=-h^{-1} W_{0}\left(-h e^{-h}\right) \quad \mathrm{e} \\
& x_{\infty}(\delta, \gamma, 1)=-\left[h W_{-1}\left(-e^{-1 / h} / h\right)\right]^{-1} .
\end{aligned}
$$

Podemos provar que, para qualquer valor de $\theta$, a seguinte desigualdade se satisfaz:

$$
x_{\infty}(\delta, \gamma, \theta)<\frac{\gamma}{\gamma+\delta}
$$

Para isto, é suficiente mostrar que $f$ e $f_{\theta}$ avaliadas no ponto $\gamma /(\gamma+\delta)$ são estritamente maiores que zero. Para $\theta \in\{0,1\}$, a fórmula (3.3) é portanto uma consequência imediata de uma desigualdade logarítimica padrão:

$$
\frac{u-1}{u}<\log u<u-1 \text { para todo } 0<u<1 .
$$


Para $\theta \in(0,1)$, usamos (3.4) para provar que a função $\theta \mapsto(\gamma /(\gamma+\delta \theta))^{1 / \theta}$ é crescente, de onde segue (3.3).

Teorema 3.2.4. Assumimos (3.1) e seja $x_{\infty}$ como na Definição 3.2.1 ou 3.2.2 de acordo a $\theta \in(0,1)$ ou não. Definimos $u_{\infty}=(1-\delta)\left(1-x_{\infty}\right)$. Então,

$$
\lim _{N \rightarrow \infty} \frac{X^{(N)}\left(\tau^{(N)}\right)}{N}=x_{\infty} e \lim _{N \rightarrow \infty} \frac{U^{(N)}\left(\tau^{(N)}\right)}{N}=u_{\infty} \quad \text { em probabilidade. }
$$

Teorema 3.2.5. Assumimos (3.1) e definimos

$$
\begin{aligned}
& \kappa=3 \theta_{1}+2 \theta_{2}-4 \gamma, \quad A=\frac{x_{\infty}}{\gamma-(\gamma+\delta) x_{\infty}}, \quad B=\frac{\gamma \delta u_{\infty}}{\gamma+\delta \theta}, \\
& C=(\gamma+\delta)^{2}\left(4 \delta \theta^{2}-\kappa(\gamma+2 \delta \theta)\right) x_{\infty}+\kappa \gamma(\gamma+\delta)(\gamma+\delta(2 \theta-1))-4 \delta \gamma^{2}(1-\theta)^{2}, \\
& D=\left\{\begin{array}{cc}
\frac{C\left(1-x_{\infty}\right)}{2(2 \theta-1)(\gamma+\delta \theta)^{2}} & \text { if } \theta \neq \frac{1}{2}, \\
\frac{2 \gamma\left[\kappa \delta(2 \gamma+\delta)-2 \gamma(\delta-\kappa(\gamma+\delta)) \log \left(\frac{\gamma}{\gamma+\delta}\right)\right]}{(\gamma+\delta)^{2}} & \text { if } \theta=\frac{1}{2} .
\end{array}\right.
\end{aligned}
$$

Então,

$$
\sqrt{N}\left(\frac{X^{(N)}\left(\tau^{(N)}\right)}{N}-x_{\infty}, \frac{U^{(N)}\left(\tau^{(N)}\right)}{N}-u_{\infty}\right) \Rightarrow N_{2}(0, \Sigma)
$$

quando $N$ tende para $\infty$, onde $N_{2}(0, \Sigma)$ é a distribuição normal bivariada com média zero e matriz de covariância $\Sigma$ dada por

$$
\begin{aligned}
& \Sigma_{11}=x_{\infty}\left(1-x_{\infty}\right)+A^{2} D, \\
& \Sigma_{12}=-(1-\delta) \Sigma_{11}+A B \\
& \Sigma_{22}=(1-\delta)^{2} \Sigma_{11}+(1-\delta)\left(\delta\left(1-x_{\infty}\right)-2 A B\right) .
\end{aligned}
$$

Quando $\delta=1$, podemos omitir a segunda componente do vetor no lado esquerdo de (3.5), e denotamos por $\sigma^{2}\left(=\Sigma_{11}\right)$ a variância da distribuição Gaussiana assintotica de média zero.

Exemplo 3.2.6. Consideremos $\rho \in[0,1]$ e seja $\lambda=\delta=\gamma=1, \theta_{1}=\rho$ e $\theta_{2}=1-\rho$. Deste modo, $\theta=0$,

$$
\begin{gathered}
x_{\infty}=x_{\infty}(1,1,0)=-\frac{W_{0}\left(-2 e^{-2}\right)}{2} \approx 0.203188, \quad \mathrm{e} \\
\sigma^{2}=\frac{x_{\infty}\left(1-x_{\infty}\right)\left(1-2 x_{\infty}+2 \rho x_{\infty}^{2}\right)}{\left(1-2 x_{\infty}\right)^{2}} \approx 0.272736+0.0379364 \rho .
\end{gathered}
$$

Obtemos o modelo [MT] ou [DK] de acordo a que $\rho$ seja 0 ou 1, mostrando que nossos teoremas generalizam resultados clássicos apresentados por Sudbury (1985) e Watson (1988).

Exemplo 3.2.7. Em um interessante ensaio, Hayes (2005) descreve um error cometido quando ele tenta simular o modelo [DK]. Na realidade, o autor simula o modelo [MT] com a diferência que quando dois informantes encontram-se ambos transformam-se em contidos. Este modelo é 
obtido escolhendo $\lambda=\delta=\gamma=1, \theta_{1}=2$ e $\theta_{2}=0$, em cujo caso $\theta=1$,

$$
\begin{gathered}
x_{\infty}=x_{\infty}(1,1,1)=-\frac{1}{2 W_{-1}\left(-e^{-1 / 2} / 2\right)} \approx 0.284668, \quad \mathrm{e} \\
\sigma^{2}=\frac{x_{\infty}\left(1-x_{\infty}\right)\left(1-3 x_{\infty}+3 x_{\infty}^{2}\right)}{\left(1-2 x_{\infty}\right)^{2}} \approx 0.427204 .
\end{gathered}
$$

Isto verifica o valor numérico da proporção de ignorantes remanescentes na população que Hayes obteve nas suas simulações.

Exemplo 3.2.8. Como já mencionamos, a variante $(\alpha, p, q)$ do modelo [DK] é obtida escolhendo (3.2). Por exemplo, se $0<\alpha(1-p)<1$, então $x_{\infty}$ é a única raiz da função

$$
f^{*}(x)=\frac{(1+q(1-p)) x^{\alpha(1-p)}-(\alpha+q)(1-p) x-1+\alpha(1-p)}{(1-p)(1-\alpha(1-p))}
$$

no intervalo $(0,1)$. Quando $q=1$, este é exatamente o valor limite obtido na análise determinística do modelo apresentada em Daley e Gani (1999, Equação (5.2.8)).

Para a variante $(\alpha, 1,1)$ do modelo $[\mathrm{DK}]$ temos que

$$
\begin{gathered}
x_{\infty}=x_{\infty}(1, \alpha, 0)=-\frac{W_{0}\left(-h e^{-h}\right)}{h} \text { com } h=1+\frac{1}{\alpha}, \mathrm{e} \\
\sigma^{2}=\frac{x_{\infty}\left(1-x_{\infty}\right)\left(2 \alpha^{2}+\left[2(1-\alpha)-\alpha(1+\alpha)^{2}\right] x_{\infty}+\alpha(1+\alpha)^{2} x_{\infty}^{2}\right)}{2\left(\alpha-(1+\alpha) x_{\infty}\right)^{2}} .
\end{gathered}
$$

Estas fórmulas coincidem com aquelas apresentadas em Daley e Gani (1999, Exercício 5.7).

Exemplo 3.2.9. Outros dois modelos de rumores que podem ser obtidos a partir de nossa cadeia de Markov geral são introduzidos em Pearce (2000) e Kawachi (2008). O primeiro segue a dinâmica do modelo $[\mathrm{DK}]$ com as seguintes regras de interação. Um encontro entre um ignorante e um informante resulta no ignorante transformando-se em informante com probabilidade $p$. Quando dois informantes interagem, ambos transformam-se em contidos com probabilidade $q_{2}$ ou só um deles o faz com probabilidade $q_{1}$. Finalmente, a interação de um informante com um contido resulta em dois contidos com probabilidade $r$. Assim, este modelo é obtido considerando

$$
\lambda=p, \quad \delta=1, \theta_{1}=q_{2} / p, \theta_{2}=q_{1} /(2 p) \text { e } \gamma=r / p .
$$

O modelo determinístico estudado em Kawachi (2008) desenvolve-se analogamente ao modelo de Hayes descrevedo no Exemplo 3.2.7. Os contatos são como no modelo [MT], mas quando dois informantes se encontram ambos transformam-se em contidos com probabilidade $\tilde{\beta}$. Quando um informante encontra um ignorante, o primeiro transmite o rumor com probabilidade $\tilde{\alpha}$ e o último se junta aos informantes com probabilidade $\tilde{\theta}$. Finalmente, em um encontro de um informante com um contido, o primeiro indivíduo transforma-se em contido com probabilidade $\tilde{\gamma}$. Este modelo é obtido da escolha

$$
\lambda=\tilde{\alpha}, \quad \delta=\tilde{\theta}, \quad \theta_{1}=\tilde{\beta} / \tilde{\alpha}, \theta_{2}=0 \text { e } \gamma=\tilde{\gamma} / \tilde{\alpha} .
$$




\subsection{Provas}

\subsubsection{Mudança do tempo}

Definimos

$$
\begin{aligned}
& \Theta^{(N)}(t)=\int_{0}^{t} Y^{(N)}(s) d s, 0 \leq t \leq \tau^{(N)}, \\
& \Upsilon^{(N)}(s)=\inf \left\{t: \Theta^{(N)}(t)>s\right\}, 0 \leq s \leq \int_{0}^{\infty} Y^{(N)}(u) d u,
\end{aligned}
$$

e seja $\tilde{V}^{(N)}(t)=V^{(N)}\left(\Upsilon^{(N)}(t)\right)$. O processo a tempo mudado $\left\{\tilde{V}^{(N)}(t)\right\}_{t \geq 0}$ tem as mesmas transições que $\left\{V^{(N)}(t)\right\}_{t \geq 0}$. Então se definimos

$$
\tilde{\tau}^{(N)}=\inf \left\{t: \tilde{Y}^{(N)}(t)=0\right\}
$$

temos que $V^{(N)}\left(\tau^{(N)}\right)=\tilde{V}^{(N)}\left(\tilde{\tau}^{(N)}\right)$. Além disso, $\left\{\tilde{V}^{(N)}(t)\right\}_{t>0}$ é uma cadeia de Markov a tempo contínuo com estado inicial $(N, 0,1)$ e transições e taxas dadas por

$$
\begin{array}{cc}
\text { transição } & \operatorname{taxa} \\
\ell_{0}=(-1,0,1) & \lambda \delta \tilde{X}, \\
\ell_{1}=(-1,1,0) & \lambda(1-\delta) \tilde{X}, \\
\ell_{2}=(0,0,-2) & \lambda \theta_{1} \frac{\tilde{Y}-1}{2}, \\
\ell_{3}=(0,0,-1) & \lambda \theta_{2}(\tilde{Y}-1)+\lambda \gamma(N+1-\tilde{X}-\tilde{Y}) .
\end{array}
$$

\subsubsection{Limite determinístico}

Definimos para $t \geq 0$,

$$
\tilde{v}^{(N)}(t)=\frac{\tilde{V}^{(N)}(t)}{N}=\left(\tilde{x}^{(N)}(t), \tilde{u}^{(N)}(t), \tilde{y}^{(N)}(t)\right)
$$

e consideramos as funções

$$
\begin{array}{ll}
\beta_{\ell_{0}}(x, u, y)=\lambda \delta x, & \beta_{\ell_{1}}(x, u, y)=\lambda(1-\delta) x \\
\beta_{\ell_{2}}(x, u, y)=\lambda \theta_{1} \frac{y}{2}, & \beta_{\ell_{3}}(x, u, y)=\lambda \theta_{2} y+\lambda \gamma(1-x-y) .
\end{array}
$$

Notemos que as taxas em (3.7) podem-se escrever como

$$
N\left[\beta_{\ell_{i}}\left(\frac{\tilde{X}}{N}, \frac{\tilde{U}}{N}, \frac{\tilde{Y}}{N}\right)+O\left(\frac{1}{N}\right)\right] .
$$

Então $\left\{\tilde{v}^{(N)}(t)\right\}_{t \geq 0}$ é uma cadeia de Markov "density dependent" com possíveis transições no conjunto $(1 / N)\left\{\ell_{0}, \ell_{1}, \ell_{2}, \ell_{3}\right\}$. 
Usamos o Teorema B.2.1 para concluir que o sistema a tempo mudado converge quasecertamente quando $N \rightarrow \infty$ (em um espaço de probabilidade adequado). A função drift é dada por

$$
F(x, u, y)=\sum_{i} \ell_{i} \beta_{\ell_{i}}(x, u, y)=(-\lambda x, \lambda(1-\delta) x, \lambda(\gamma+\delta) x-\lambda \theta y-\lambda \gamma),
$$

e portanto o sistema determinístico limite é governado pelo sistema de equações diferenciais ordinárias

$$
\left\{\begin{array}{l}
x^{\prime}(t)=-\lambda x(t), \\
u^{\prime}(t)=\lambda(1-\delta) x(t), \\
y^{\prime}(t)=\lambda(\gamma+\delta) x(t)-\lambda \theta y(t)-\lambda \gamma,
\end{array}\right.
$$

com condições iniciais $x(0)=1, u(0)=0$ e $y(0)=0$. A solução deste sistema é dada por $v(t)=(x(t), u(t), y(t))$, onde

$$
\begin{gathered}
x(t)=e^{-\lambda t}, \\
u(t)=(1-\delta)(1-x(t)), \quad \mathrm{e} \\
y(t)=f(x(t)),
\end{gathered}
$$

substituindo $f$ por $f_{\theta}$ se $\theta$ é igual a 0 ou 1 .

De acordo ao Teorema B.2.1, temos que

$$
\lim _{N \rightarrow \infty} \tilde{v}^{(N)}(t)=v(t) \quad \text { q. c. }
$$

uniformemente em intervalos de tempo limitados. Em particular, provamos que para cada uma das dois primeiras coordenadas de $\tilde{v}^{(N)}$, a convergência é uniforme em toda a reta.

Lema 3.3.1. Vale que

$$
\lim _{N \rightarrow \infty} \tilde{x}^{(N)}(t)=x(t) \quad \text { q. c. },
$$

uniformemente em $\mathbb{R}$. Uma afirmação análoga vale para $\tilde{u}^{(N)}(t)$ e $u(t)$.

Demonstração. Provamos a primeira afirmação. Dado qualquer $\varepsilon>0$, tomamos $t_{0}=t_{0}(\varepsilon)$ tal que $x(t) \leq \varepsilon / 2$ para todo $t \geq t_{0}$. Pela convergência uniforme no intervalo $\left[0, t_{0}\right]$, existe $N_{0}=N_{0}(\varepsilon)$ tal que

$$
\left|\tilde{x}^{(N)}(t)-x(t)\right| \leq \varepsilon / 2 \text { para todo } N \geq N_{0} \text { e } t \in\left[0, t_{0}\right]
$$

Logo, para todo $N \geq N_{0}$ e $t \geq t_{0}$,

$$
\tilde{x}^{(N)}(t) \leq \tilde{x}^{(N)}\left(t_{0}\right) \leq x\left(t_{0}\right)+\varepsilon / 2 \leq \varepsilon,
$$

quando $\left|\tilde{x}^{(N)}(t)-x(t)\right| \leq \varepsilon$. 


\subsubsection{Provas dos teoremas}

Ambos teoremas seguem do Teorema B.3.2. Adoptamos as notações apresentadas no apêndice, sendo neste caso $\mathcal{V}=\left(\mathcal{V}_{x}, \mathcal{V}_{u}, \mathcal{V}_{y}\right)$. Aqui $\varphi(x, u, y)=y$, e

$$
\tau_{\infty}=\inf \{t: y(t) \leq 0\}=-\frac{1}{\lambda} \log x_{\infty}
$$

Logo, de (3.3),

$$
\nabla \varphi\left(v\left(\tau_{\infty}\right)\right) \cdot F\left(v\left(\tau_{\infty}\right)\right)=y^{\prime}\left(\tau_{\infty}\right)=\lambda(\gamma+\delta) x_{\infty}-\lambda \gamma<0
$$

Vamos descrever o argumento para a prova da Lei dos Grandes Números. Embora $y(0)=0$, temos que $y^{\prime}(0)>0$. Isto e (2.14) implicam que $y\left(\tau_{\infty}-\varepsilon\right)>0$ e $y\left(\tau_{\infty}+\varepsilon\right)<0$ para $0<\varepsilon<\tau_{\infty}$. A convergência quase certa de $\tilde{y}^{(N)}$ para $y$ uniformemente em intervalos limitados implica que

$$
\lim _{N \rightarrow \infty} \tilde{\tau}^{(N)}=\tau_{\infty} \quad \text { quase certamente. }
$$

Desta maneira, lembrando que $V^{(N)}\left(\tau^{(N)}\right)=\tilde{V}^{(N)}\left(\tilde{\tau}^{(N)}\right)$, obtemos o Teorema 3.2.4 da fórmula (3.9) e o Lema 3.3.1.

Com relação ao Teorema Central do Limite, obtemos do Teorema B.3.2 que

$$
\sqrt{N}\left(\tilde{x}^{(N)}\left(\tilde{\tau}^{(N)}\right)-x_{\infty}, \tilde{u}^{(N)}\left(\tilde{\tau}^{(N)}\right)-u_{\infty}\right)
$$

converge em distribuição quando $N \rightarrow \infty$ para

$$
\left(\mathcal{V}_{x}\left(\tau_{\infty}\right)-A \mathcal{V}_{y}\left(\tau_{\infty}\right), \mathcal{V}_{u}\left(\tau_{\infty}\right)+A(1-\delta) \mathcal{V}_{y}\left(\tau_{\infty}\right)\right)
$$

onde $A$ é a constante definida no Teorema 3.2.5. A distribuição assintotica é uma distribuição normal bivariada com média zero, então só falta mostrar como é obtida a fórmula (3.6) para a matriz de covariâncias $\Sigma$.

Para isto, enumeramos os passos seguidos para calcular $\Lambda=\operatorname{Cov}\left(\mathcal{V}\left(\tau_{\infty}\right), \mathcal{V}\left(\tau_{\infty}\right)\right)$. Primeiro, calculamos a matriz de derivadas parciais da função drift $F$ e a matriz $G$, como definidas no apêndice. Estas são dadas por

$$
\begin{gathered}
\partial F(x, u, y)=\left(\begin{array}{ccc}
-\lambda & 0 & 0 \\
\lambda(1-\delta) & 0 & 0 \\
\lambda(\gamma+\delta) & 0 & -\lambda \theta
\end{array}\right) \mathrm{e} \\
G(x, u, y)=\left(\begin{array}{ccc}
\lambda x & -\lambda(1-\delta) x & -\lambda \delta x \\
-\lambda(1-\delta) x & \lambda(1-\delta) x & 0 \\
-\lambda \delta x & 0 & \lambda(\delta-\gamma) x+\lambda(\kappa-\theta+2 \gamma) y+\lambda \gamma
\end{array}\right) .
\end{gathered}
$$


Logo, obtemos a solução $\Phi$ da equação matricial

$$
\frac{\partial}{\partial t} \Phi(t, s)=\partial F(x(t), u(t), y(t)) \Phi(t, s), \quad \Phi(s, s)=\mathbb{I}_{3} .
$$

Então, calculamos

$$
\operatorname{Cov}(\mathcal{V}(t), \mathcal{V}(t))=\int_{0}^{t} \Phi(t, s) G(x(s), u(s), y(s))[\Phi(t, s)]^{T} d s
$$

Vale enfatizar que o cálculo de $\Lambda$ pode ser separado em quatro casos: $\theta \in(0,1) \backslash\{1 / 2\}$, $\theta=1 / 2, \theta=0$ e $\theta=1$. O valor $\theta=1 / 2$ precisa ser considerado separadamente do intervalo $(0,1)$ dada a ocorrência da integral $\int_{0}^{t} e^{\lambda(2 \theta-1) s} d s$ no elemento $(3,3)$ da matriz dada na fórmula (3.11). Isto alias explica porque a constante $D$ no Teorema 2.2.5 é definida diferentemente para $\theta=1 / 2$.

Obviamente, o passo final para obter $\Lambda$ é tomar $t=\tau_{\infty}$ na fórmula obtida de (3.11). Isto é conseguido fazendo substituições convenientes nesta fórmula de acordo ao valor de $\theta$; por exemplo, para $\theta \in(0,1) \backslash\{1 / 2\}$ substituimos $e^{-\lambda t}$ e $e^{-\lambda \theta t}$ respectivamente por

$$
x_{\infty} \text { e } \frac{(\gamma+\delta) \theta x_{\infty}+\gamma(1-\theta)}{\gamma+\delta \theta} .
$$

A fórmula resultante (válida para qualquer $\theta$ ) é

$$
\Lambda=\left(\begin{array}{ccc}
x_{\infty}\left(1-x_{\infty}\right) & -(1-\delta)\left(1-x_{\infty}\right) x_{\infty} & 0 \\
-(1-\delta)\left(1-x_{\infty}\right) x_{\infty} & (1-\delta)\left(1-x_{\infty}\right)\left((1-\delta) x_{\infty}+\delta\right) & -B \\
0 & -B & D
\end{array}\right)
$$

Usando (3.10), (3.12) e propriedades bem conhecidas da variância e a covariância, conseguimos a fórmula (3.6). 


\section{Capítulo 4}

\section{Uma abordagem via sistemas de partículas}

Neste capítulo interpretamos a difusão de um rumor como um sistema de passeios aleatórios em um grafo. Em particular, mostramos que uma simples variante do modelo estudado em Kurtz et al. (2008) é suficiente para descrever a versão $k$-fold stifling do modelo [MT] apresentada em Belen (2008).

\subsection{O modelo [ALMM]}

No modelo introduzido em Alves et al. (2006), chamado na sequência de modelo [ALMM], considera-se um sistema de passeios aleatórios no grafo completo. No tempo zero existe em cada vértice do grafo uma partícula que pode ser ou ativa ou inativa. Cada partícula ativa começa realizar um passeio aleatório pelos vértices do grafo seguindo as seguintes regras:

1. Quando uma partícula ativa entra em contato com uma partícula inativa, esta última é ativada e começa realizar, de maneira independente, um passeio aleatório pelo grafo;

2. as partículas ativas podem visitar qualquer vértice do grafo;

3. cada partícula ativa morre no instante em que faz um salto sem ativar nenhuma partícula.

Este modelo, formulado incialmente a tempo discreto, é uma variante do "modelos dos sapos" bem estudado em grafos infinitos em Alves et al. (2002a,b) e Lebensztayn et al. (2005), entre outros. Para uma revisão deste tipo de modelos sugerimos consultar Popov (2003).

Uma quantidade de interesse para o modelo [ALMM] é a proporção de sítios visitados ao final do processo, isto é, quando não há mais partículas ativas. Esta proporção é estudada pela primeira vez em Alves et al. (2006) por meio de simulações e uma análise de campo médio do processo.

Kurtz et al. (2008) introduzem uma variante a tempo contínuo, que chamamos de versão $k$-vidas do modelo [ALMM], onde assume-se que cada partícula ativa morre no instante em que 
faz um número finito $k$ de saltos sem ativar nenhuma partícula. Neste caso, provam-se teoremas limites para a proporção de sítios visitados ao final do processo.

Nosso objetivo é comparar a versão $k$-vidas do modelo [ALMM] com um processo de difusão de um rumor. Para isto, introduzimos um modelo auxiliar que conduze-nos aos mesmos resultados obtidos em Kurtz et al. (2008) e que pode ser acoplado à versão $k$-fold stifling do modelo $[\mathrm{MT}]$.

\subsubsection{O modelo auxiliar}

Consideramos a seguinte variante da versão $k$-vidas do modelo [ALMM], chamada de modelo auxiliar na sequencia. No tempo zero existe em cada vértice do grafo uma partícula ativa ou inativa, e cada partícula ativa começa realizar um passeio aleatório simétrico a tempo contínuo pelos vértices do grafo seguindo as seguintes regras:

1. Quando uma partícula ativa entra em contato com uma partícula inativa, esta última é ativada e começa realizar, de maneira independente, um passeio aleatório pelo grafo;

2. nenhuma partícula ativa pode visitar novamente o vértice donde ela começou o passeio;

3. cada partícula ativa morre no instante em que faz um número finito $k$ de saltos sem ativar nenhuma partícula.

Formalmente, consideramos o grafo completo de $N+1$ vértices e usando a notação de Kurtz et al. (2008) denotamos ao número de sítios visitados e de partículas ativas com $i$ vidas no instante $t$ por $V^{(N)}(t)$ e $A_{i}^{(N)}(t)$ respectivamente, para $i=1, \ldots, k$. Quando nos referimos ao número de vidas de uma partícula queremos dizer o número de vezes que ela passara por um sítio anteriormente visitado, por alguma partícula ativa, antes de morrer.

Assim definido, o processo

$$
\left\{\left(V^{(N)}(t), A_{1}^{(N)}(t), \ldots, A_{k}^{(N)}(t)\right)\right\}_{t \geq 0}
$$

é uma cadeia de Markov a tempo contínuo com transições e correspondentes taxas dadas por

$$
\begin{array}{cc}
\text { transição } & \text { taxa } \\
(1,0, \ldots, 0,0, \ldots, 1) & (N-V+1) \sum_{i=1}^{k} A_{i}, \\
(0,0, \ldots, 1,-1, \ldots, 0) & (V-1) A_{i}, \quad \text { para } i=2, \ldots, k \\
(0,-1, \ldots, 0,0, \ldots, 0) & (V-1) A_{1} .
\end{array}
$$

Em palavras, a primeira transição indica o evento no qual uma partícula inativa é ativada; a segunda indica o evento que uma partícula ativa com $i$ vidas perde uma vida (para $i=2, \ldots, k$ ) e a última representa a morte de uma partícula ativa com uma vida.

Aplicando as mesmas idéias usadas em Kurtz et al. (2008) pode-se concluir que o modelo auxiliar proposto tem o mesmo comportamento limite que a versão $k$-vidas do modelo [ALMM] 
e portanto valem os mesmos teoremas limites. Em particular, se assumimos que $V^{(N)}(0)=N$ e $A_{k}^{(N)}(0)=1$, então obtemos que

$$
\lim _{N \rightarrow \infty} \frac{V^{(N)}\left(T^{(N)}\right)}{N}=v_{\infty} \quad \text { em probabilidade }
$$

onde $T^{(N)}$ é o tempo de absorção do processo e

$$
v_{\infty}=1+(k+1)^{-1} W_{0}\left(-(k+1) e^{-(k+1)}\right)
$$

é a quantidade obtida em Kurtz et al. (2008). No entanto, como naquele trabalho, este resultado pode ser obtido para condições inciais gerais.

Na próxima seção mostramos como este modelo auxiliar é suficiente para descrever a versão $k$-fold stifling do modelo [MT].

\subsection{A difusão de um rumor como um sistema de partículas}

Na versão $k$-fold stifling do modelo [MT] assume-se que um informante só decide parar de transmitir o rumor no instante em que encontra-se com o $k$-ésimo indivíduo já informado, seja este informante ou contido. Na Observação 2.2.7 notamos que este modelo resulta de considerar $R \equiv k$ na variante random stifling do modelo [MT] proposta no Capítulo 2. Mantendo a notação desse capítulo temos que no instante $t, X^{(N)}(t), Y_{i}^{(N)}(t)$ e $Z^{(N)}(t)$ representam o número de ignorantes, informantes de tipo $i$ e contidos, respectivamente, sendo que neste caso $i=1, \ldots, k$. O processo $k$-dimensional

$$
\left\{\left(X^{(N)}(t), Y_{1}^{(N)}(t), \ldots, Y_{k}^{(N)}(t)\right)\right\}_{t \geq 0}
$$

é uma cadeia de Markov a tempo contínuo que, de acordo a (2.2) para o caso $R \equiv k$, tem transições e correspondentes taxas dadas por

$$
\begin{array}{cc}
\text { transição } & \text { taxa } \\
(-1,0, \ldots, 0,0, \ldots, 1) & X \sum_{i=1}^{k} Y_{i}^{(N)}, \\
(0,0, \ldots, 1,-1, \ldots, 0) & (N-X) Y_{i}, \quad \text { para } i=2, \ldots, k \\
(0,-1, \ldots, 0,0, \ldots, 0) & (N-X) Y_{1} .
\end{array}
$$

Notemos que estas transições e taxas são semelhantes às obtidas na seção anterior. Esta semelhança fica em evidência depois de representar a versão $k$-fold stifling do modelo [MT] como o modelo auxiliar definido na seção precedente. Mais precisamente, consideramos:

1. o grafo completo de $N+1$ vértices para representar a população misturada, homogênea e fechada de $N+1$ indivíduos, e

2. o sistema de passeios aleatórios para representar a difusão do rumor na população. 
Nesta representação cada partícula ativa está associada a um informante e cada partícula inativa a um ignorante. Esta relação fica determinada no tempo zero onde há uma partícula por cada vértice. Logo, com a posição de uma partícula ativa em um instante de tempo determinado indica-se o indivíduo com o qual o informante que ela representa esta tendo um contato. Diante disso, quando uma partícula ativa morre entende-se que o informante ao qual ela representa transforma-se em contido. Com efeito, lembremos que uma partícula ativa morre depois de visitar $k$ sitios anteriormente visitados por alguma partícula ativa. Mas isto é equivalente ao evento em que o informante ao qual esta partícula esta associada tem $k$ contatos com indivíduos já informados do rumor.

Segue-se que com esta representação podemos construir ambos processos em um mesmo espaço de probabilidade e de forma tal que o número de sitios visitados por uma partícula ativa em um instante de tempo $t$ é igualmente distribuído ao número de indivíduos informados na população até esse momento. Isto é, de maneira que

$$
V^{(N)}(t)=N+1-X^{(N)}(t)
$$

em distribuição. Deste modo, concluimos de (4.3) e (4.1) que a proporção de ignorantes ao final do processo converge em probabilidade para

$$
1-v_{\infty}=-(k+1)^{-1} W_{0}\left(-(k+1) e^{-(k+1)}\right)
$$

que é exatamente o valor obtido para o análogo determinístico do modelo em Belen (2008).

\subsection{Comentários finais}

Neste capítulo discutimos a relação entre dois modelos que, formulados de maneira independente e em diferentes contextos, podem ser pensados como um em função do outro. Para isto, introduzimos um modelo auxiliar com o mesmo comportamento limite que a versão $k$-vidas do modelo $[\mathrm{ALMM}]$ e que pode ser acoplado à versão $k$-fold stifling do modelo [MT]. Para este último, Belen (2008) encontra a proporção final de ignorantes para a versão determinística e para condições iniciais específicas, isto é, um informante e $N$ ignorantes. No entanto, os resultados de Kurtz et al. (2008) e nossa comparação dos modelos permitem-nos estabelecer uma lei dos grandes números e um teorema central do limite desta proporção na versão estocástica. Mais ainda, isto pode ser feito para condições iniciais gerais.

Das observações anteriores concluimos que o modelo [ALMM] pode ser uma ótima ferramenta para descrever diferentes processos da difussão de um rumor ou uma informação. Como um outro exemplo disto, mencionamos uma generalização natural da versão $k$-vidas do modelo [ALMM] que surge de tomar o $k$ como sendo uma variável aleatória. O mesmo raciocinio usado neste capítulo permite-nos concluir que esta generalização é adequada para descrever a variante random stifling do modelo [MT] introduzida no Capítulo 2.

Por outro lado, considerar o modelo [ALMM] em outro tipo de grafos finitos pode nos conduzir a uma melhor compreensão de diferentes processos reais de difusão de uma informação. 


\section{Apêndice A}

\section{Construção gráfica dos modelos}

No que segue apresentamos uma construção gráfica dos modelos [DK] e [MT].

\section{A.1 Construção do modelo [DK]}

Consideramos o grafo completo de $N+1$ vértices que denotamos por $K_{N+1}$ e cujo conjunto de vértices denotamos por $[N+1]:=\{1, \ldots, N+1\}$. Dizemos que cada vértice do grafo esta em um dos estados: 0 (ignorante), 1 (informante) e 2 (contido). Denotamos ao processo por $\xi_{t} \in\{0,1,2\}^{[N+1]}$ e notamos que a cadeia $\{(X(t), Y(t))\}_{t \geq 0}$ definida na Seção 1.3 pode ser obtida como

$$
\begin{aligned}
X(t) & =\sum_{v \in[N+1]} I_{\left\{\xi_{t}(v)=0\right\}} \mathrm{e} \\
Y(t) & =\sum_{v \in[N+1]} I_{\left\{\xi_{t}(v)=1\right\}} .
\end{aligned}
$$

Daremos uma construção gráfica para o processo $\xi_{t}$. Para isto, consideramos uma família de processos de Poisson independentes de taxa $1,\left\{L^{\{u, v\}}: u, v \in[N+1]\right\}$, onde $\{u, v\}$ denota ao elo entre os vértices $u$ e $v$.

Para a construção consideramos a região espaço-temporal $K_{N+1} \times(0, \infty)$. Associamos o processo $L^{\{u, v\}}$ ao elo $\{u, v\}$ e em cada chegada de um ponto deste processo fazemos as seguintes trocas de estado:

- Se $u$ (ou $v$ ) está no estado 0 e $v$ (ou $u$ ) está no estado 1: $u$ (ou $v$ ) troca para o estado 1,

- se $u$ (ou $v$ ) está no estado 1 e $v$ (ou $u$ ) está no estado 2: $u$ (ou $v$ ) troca para o estado 2,

- se ambos $u$ e $v$ estão no estado 1: ambos cambiam ao estado 2,

- nada acontece em qualquer outro caso.

Desta forma obtemos uma versão do processo estocástico $\xi_{t}$ e portanto da cadeia $\{(X(t), Y(t))\}_{t \geq 0}$ definida na Seção 1.3 com taxas dadas por (1.1). Observamos que consideramos processos de Poisson de taxa 1 sem perda de generalidade pois o processo pode ser definido com processos de Poisson com taxa $\lambda>0$ mudando com isto a velocidade do processo. 


\section{A.2 Construção do modelo [MT]}

Para a construção do modelo [MT] seguimos os mesmos passos da seção anterior. Neste caso consideramos o grafo completo direcionado, com um elo direcionado entre quaisquer dois vértices distintos. Denotamos ao grafo por $\vec{K}_{N+1}$ e ao conjunto de vértices por $[N+1]$. Dizemos que cada vértice do grafo está em um dos estados: 0, 1 e 2. Denotamos a este processo por $\eta_{t} \in\{0,1,2\}^{[N+1]}$ e observamos que podemos definir a cadeia $\{(X(t), Y(t))\}_{t \geq 0}$ da Seção 1.4 como

$$
\begin{aligned}
X(t) & =\sum_{v \in[N+1]} I_{\left\{\eta_{t}(v)=0\right\}} \mathrm{e} \\
Y(t) & =\sum_{v \in[N+1]} I_{\left\{\eta_{t}(v)=1\right\}} .
\end{aligned}
$$

Para a construção gráfica do processo $\eta_{t}$, consideramos uma família de processos de Poisson independentes de taxa $1,\left\{L^{(u, v)}:(u, v) \in[N+1] \times[N+1]\right.$ e $\left.u \neq v\right\}$. Notemos que neste caso, a diferência da construção anterior, temos dois processos de Poisson associados para cada par de vértices. Podemos representar isto na região espaço-temporal $\vec{K}_{N+1} \times(0, \infty)$ e associando os pontos do processo $L^{(u, v)}$ ao elo direcionado no sentido de $u$ para $v$. Desta maneira, cada chegada de um ponto de $L^{(u, v)}$ representa as seguintes trocas de estado:

- Se $u$ está no estado 1 e $v$ está no estado 0: $v$ troca para o estado 1 ,

- se $u$ está no estado 1 e $v$ está no estado 1 ou 2: $u$ troca para o estado 2,

- nada acontece em qualquer outro caso.

Desta forma obtemos uma versão do processo estocástico $\eta_{t}$ e portanto da cadeia $\{(X(t), Y(t))\}_{t \geq 0}$ definida na Seção 1.4 com taxas dadas por (1.2). 


\section{Apêndice B}

\section{Cadeias de Markov "density dependent"}

Enunciamos os principais conceitos e teoremas de convergência que são aplicados ao longo da tese, referentes à teoria das cadeias de Markov "density dependent". Como se trata de uma teoria muito citada na literatura, faremos uma abordagem rápida dos resultados. Para mais detalhes o leitor pode consultar o livro de Andersson e Britton (2000) (Capítulo 5) ou Ethier e Kurtz (2005) (Capítulo 11).

\section{B.1 Definição e caracterização do processo}

Para cada $n \geq 1$, seja $Z_{n}=\left(Z_{n}(t)\right)_{t \geq 0}$ uma cadeia de Markov a tempo contínuo no espaço de estados $d$-dimensional $\mathbb{Z}^{d}$ governado pelas taxas de transição

$$
q_{z, z+\ell}^{(n)}=n \beta_{\ell}\left(\frac{z}{n}\right), \quad z, \ell \in \mathbb{Z}^{d}
$$

Isto é,

$$
\begin{array}{r}
P\left(Z_{n}(t+h)=z+\ell \mid Z_{n}(t)=z\right)=h n \beta_{\ell}\left(\frac{z}{n}\right)+o(h), \quad \ell \neq 0 \\
P\left(Z_{n}(t+h)=z \mid Z_{n}(t)=z\right)=1-h n \sum_{\ell} \beta_{\ell}\left(\frac{z}{n}\right)+o(h) .
\end{array}
$$

Notemos que assim definidas as taxas dependen da "densidade" do processo. Esta é a justificação do nome de "density dependent" dado à cadeia de Markov $Z_{n}$.

Supomos que o processo tem no máximo um número enumerável de possíveis transições e que as funções $\beta_{\ell}(x)$ são continuas. Além disto, supomos que o estado inicial do processo $Z_{n}(0)$ é não aleatório.

Uma maneira de caracterizar o processo $Z_{n}$ é por medio de processos de Poisson. Com efeito, se consideramos processos de Poisson independentes de taxa $1, Y_{\ell}=\left(Y_{\ell}(t)\right)_{t \geq 0}$, definidos para cada uma das possíveis transições $\ell$, podemos escrever

$$
Z_{n}(t)=Z_{n}(0)+\sum_{\ell} \ell Y_{\ell}\left(n \int_{0}^{t} \beta_{\ell}\left(\frac{Z_{n}(s)}{n}\right) d s\right) .
$$

Para verificar que (B.2) satisfaz (B.1) notemos que dado $Z_{n}(t)=z$, a probabilidade que haverá 
uma transição em

$$
Y_{\ell}\left(n \int_{0}^{t} \beta_{\ell}\left(\frac{Z_{n}(s)}{n}\right) d s\right)
$$

durante $(t, t+h)$ é $h n \beta_{\ell}\left(\frac{z}{n}\right)+o(h)$ pois o integrando é igual a $n \beta_{\ell}\left(\frac{z}{n}\right)$ até o primeiro salto depois de $t$. Observemos que os processos são definidos no mesmo espaço de probabilidade para diferentes valores de $n$, pois na construção usamos os mesmos processos de Poisson. No que segue mostraremos teoremas limites para os processos $\left\{Z_{n}\right\}$.

\section{B.2 Lei dos Grandes Números}

Para formular uma Lei dos Grandes Números definimos a função drift $F$ por

$$
F(x)=\sum_{\ell} \ell \beta_{\ell}(x), \quad x \in \mathbb{Z}^{d}
$$

e consideramos o processo $\bar{Z}_{n}(t)=n^{-1} Z_{n}(t)$. Deste modo, a equação (B.2) é equivalente a

$$
\bar{Z}_{n}(t)=\bar{Z}_{n}(0)+\sum_{\ell} \ell n^{-1} \tilde{Y}_{\ell}\left(n \int_{0}^{t} \beta_{\ell}\left(\bar{Z}_{n}(s)\right) d s\right)+\int_{0}^{t} F\left(\bar{Z}_{n}(s)\right) d s
$$

onde $\tilde{Y}_{\ell}(t)=Y_{\ell}(t)-t$ é o processo Poisson centrado na sua média. Supondo que o número de possíveis transições do processo é finito e observando que

$$
\lim _{n \rightarrow \infty} \sup _{s \leq t}\left|\frac{\tilde{Y}_{\ell}(n s)}{n}\right|=0 \quad \text { q.c., } \quad t \geq 0
$$

é fácil ver que o segundo termo do lado direto de (B.4) será pequeno para $n$ suficientemente grande. Isto sugere que $\bar{Z}_{n}(t)$ se assemelha à função vetorial determinística $z(t)$ definida como a solução da equação integral

$$
z(t)=z(0)+\int_{0}^{t} F(z(s)) d s
$$

Esta ideia é formalizada no seguinte teorema e vale para no máximo um número enumerável de possíveis transições do processo.

Teorema B.2.1. Supomos que $\lim _{n \rightarrow \infty} \bar{Z}_{n}(0)=z_{0}$ e que para cada compacto $K \subset \mathbb{R}^{d}$,

$$
\sum_{\ell}|\ell| \sup _{x \in K} \beta_{\ell}(x)<\infty
$$

e que existe uma constante $M_{K}>0$ tal que

$$
|F(x)-F(y)| \leq M_{K}|x-y|, \quad \text { para todo } x, y \in K \text {. }
$$


Então, para todo $t \geq 0$,

$$
\lim _{n \rightarrow \infty} \sup _{s \leq t}\left|\bar{Z}_{n}(s)-z(s)\right|=0, \quad \text { q.c. },
$$

onde $z(t)$ é a única solução para (B.5).

Demonstração. Ver Teorema 11.2.1 de Ethier e Kurtz (2005).

\section{B.3 Teorema Central do Limite}

Se definimos

$$
W_{\ell}^{n}(t)=\sqrt{n}\left(n^{-1} Y_{\ell}(n t)-t\right)=n^{-1 / 2} \tilde{Y}_{\ell}(n t)
$$

sabemos que $W_{\ell}^{n}$ converge para o movimento Browniano padrão $W_{\ell}$. Então,

$$
\begin{aligned}
\mathcal{V}_{n}(t): & =\sqrt{n}\left(\bar{Z}_{n}(t)-z(t)\right) \\
& =\mathcal{V}_{n}(0)+\sum_{\ell} \ell W_{\ell}^{n}\left(\int_{0}^{t} \beta_{\ell}\left(\bar{Z}_{n}(s)\right) d s\right)+\int_{0}^{t} \sqrt{n}\left(F\left(\bar{Z}_{n}(s)\right)-F(z(s))\right) d s .
\end{aligned}
$$

Notemos que $\mathcal{V}_{n}(0)=\sqrt{n}\left(\bar{Z}_{n}(0)-z(0)\right)$ em (B.7) é por hipotese não aleatório. A segunda igualdade é consequência direta da definição de $W_{\ell}^{n}, \bar{Z}_{n}$ e $z$. Por outro lado, temos que

$$
\begin{aligned}
\sqrt{n}\left(F\left(\bar{Z}_{n}(s)\right)-F(z(s))\right) & =\sqrt{n} \partial F(z(s))\left(\bar{Z}_{n}(s)-z(s)\right)+o\left(\sqrt{n}\left|\bar{Z}_{n}(s)-z(s)\right|^{2}\right) \\
& =\partial F(z(s)) \mathcal{V}_{n}(s)+o\left(\left|\bar{Z}_{n}(s)-z(s)\right|\right) \mathcal{V}_{n}(s)
\end{aligned}
$$

onde $\partial F=\left(\partial_{j} F_{i}\right)$ é a função matricial de derivadas parciais. Do Teorema B.2.1 sabemos que $\bar{Z}_{n}$ converge para $z$, e que $W_{\ell}^{n}$ converge para $W_{\ell}$, um movimento Browniano padrão. Isto sugere que $\mathcal{V}_{n}$ converge para um processo $\mathcal{V}$ definido pela equação integral

$$
\mathcal{V}(t)=\mathcal{V}(0)+\sum_{\ell} \ell W_{\ell}^{n}\left(\int_{0}^{t} \beta_{\ell}(z(s)) d s\right)+\int_{0}^{t} \partial F(z(s)) \mathcal{V}(s) d s .
$$

Isto é formalizado no seguinte teorema.

Teorema B.3.1. Supomos que para cada compacto $K \subset \mathbb{R}^{d}$,

$$
\sum_{\ell}|\ell|^{2} \sup _{x \in K} \beta_{\ell}(x)<\infty
$$

e que $\partial F$ é contínua. Supomos que

$$
\lim _{n \rightarrow \infty} \mathcal{V}_{n}(0)=\mathcal{V}(0) \quad \text { (constante) }
$$

Então

$$
\mathcal{V}_{n} \Rightarrow \mathcal{V}
$$

onde $\Rightarrow$ denota convergência em distribuição e $\mathcal{V}$ é o processo definido na equação (B.8). Este 
processo $\mathcal{V}$ é um processo vectorial Gaussiano com matriz de covariância

$$
\operatorname{Cov}(\mathcal{V}(t), \mathcal{V}(r))=\int_{0}^{r \wedge t} \Phi(t, s) G(z(s))[\Phi(r, s)]^{T} d s
$$

onde $\Phi$ e a função matricial definida como a solução de

$$
\frac{\partial}{\partial t} \Phi(t, s)=\partial F(z(t)) \Phi(t, s), \quad \Phi(s, s)=\mathbb{I}
$$

$e G(x)=\sum_{\ell} \ell \ell^{T} \beta_{\ell}(x)$

Demonstração. Ver Teorema 11.2.3 de Ethier e Kurtz (2005).

Mais ainda, pode-se provar o seguinte teorema que será de nosso interese.

Teorema B.3.2. Seja $\varphi$ continuamente diferenciável em $\mathbb{R}^{d}$. Sejam $\bar{Z}_{n}$ e z definidos como antes, com $\varphi(z(0))>0$, e supomos que as condiões do Teorema B.3.1 são satisfeitas. Seja,

$$
\tau_{n}=\inf \left\{t: \varphi\left(\bar{Z}_{n}(t)\right) \leq 0\right\}
$$

$e$

$$
\tau=\inf \{t: \varphi(z(t)) \leq 0\}
$$

Supomos que $\tau<\infty$ e

$$
\nabla \varphi(z(\tau)) \cdot F(z(\tau))<0
$$

Então,

$$
\sqrt{n}\left(\tau_{n}-\tau\right) \Rightarrow-\frac{\nabla \varphi(z(\tau)) \cdot \mathcal{V}(\tau)}{\nabla \varphi(z(\tau)) \cdot F(z(\tau))}
$$

$e$

$$
\sqrt{n}\left(\bar{Z}_{n}\left(\tau_{n}\right)-z(\tau)\right) \Rightarrow \mathcal{V}(\tau)-\frac{\nabla \varphi(z(\tau)) \cdot \mathcal{V}(\tau)}{\nabla \varphi(z(\tau)) \cdot F(z(\tau))} F(z(\tau))
$$

Demonstração. Ver Teorema 11.4.1 de Ethier e Kurtz (2005). 


\section{Referências Bibliográficas}

O. S. M. Alves, E. Lebensztayn, F. P. Machado e M. Z. Martinez. Random walks systems on complete graphs. Bull. Braz. Math. Soc., 37 (4):571-580, 2006. Citado na pág. 28

O. S. M. Alves, F. P. Machado e S Yu. Popov. Phase transition for the frog model. Electron. J. Probab., 7 (16):1-21, 2002a. Citado na pág. 28

O. S. M. Alves, F. P. Machado e S. Yu. Popov. The shape theorem for the frog model. Ann. Appl. Probab., 12 (2):533-546, 2002b. Citado na pág. 28

H. Andersson e T. Britton. Stochastic epidemic models and their statistical analysis. Springer Verlag, New York, 2000. Lecture Notes in Statistics, Vol. 151. Citado na pág. 2, 34

S. Belen. The behaviour of stochastic rumours. PhD thesis, School of Mathematical Sciences, The University of Adelaide, Austrália, Julho 2008. Citado na pág. 6, 13, 28, 31

S. Belen e C. E. M. Pearce. Rumours with random initial conditions. ANZIAM J., 45:393-400, 2004. Citado na pág. 2, 6, 13

H. Carnal. Calcul des probabilités et modélisation. El. Math., 49 (4):168-173, 1994. Citado na pág. $2,6,13$

R. M. Corless, G. H. Gonnet, D. E. G. Hare, D. J. Jeffrey e D. E. Knuth. On the lambert w function. Adv. Comput. Math., 5 (4):329-359, 1996. Citado na pág. 10

D. J. Daley e J. Gani. Epidemic Modelling: an Introduction. Cambridge University Press, Cambridge, 1999. Citado na pág. 2, 5, 7, 19, 23

D. J. Daley e D. G. Kendall. Stochastic rumours. J. Inst. Math. Appl., 1:42-55, 1965. Citado na pág. $1,4,5$

K. Dietz. Epidemics and rumours: a survey. J. Roy. Statist. Soc. Ser. A, 130 (4):505-528, 1967. Citado na pág. 2

S. N. Ethier e T. G. Kurtz. Markov Process: Characterization and Convergence. John Wiley \& Sons Inc., New York, 2005. Wiley Series in Probability and Mathematical Statistics. Citado na pág. $2,15,34,36,37$

W. Feller. An Introduction to Probability Theory and its Applications, Vol. 1. John Wiley and Sons, New York, segunda edition, 1957. Citado na pág. 1

J. Gani. The maki-thompson rumour model: a detailed analysis. Environ. Modelling Software, 15:721-725, 2000. Citado na pág. 2

W. Goffman e V. A. Newill. Generalization of epidemic theory: an application to the transmission of ideas. Nature, 204 (4955):225-228, 1964. Citado na pág. 1 
B. Hayes. Rumours and errours. American Scientist, 93:207-211, 2005. Citado na pág. 22

K. Kawachi. Deterministic models for rumor transmission. Nonlinear Anal. Real World Appl., 9:1989-2028, 2008. Citado na pág. 2, 23

M. Kosfeld. Rumours and markets. J. Math. Econom., 41:646-664, 2005. Citado na pág. 1

T. G. Kurtz, E. Lebensztayn, A. R. Leichsenring e F. P. Machado. Limit theorems for an epidemic model on the complete graph. Alea, 4:45-55, 2008. Citado na pág. 28, 29, 30, 31

E. Lebensztayn, F. P. Machado e S. Popov. An improved upper bound for the critical probability of the frog model on homogeneous trees. J. Satist. Phys., 119 (1-2):331-345, 2005. Citado na pág. 28

E. Lebensztayn, F. P. Machado e P. M. Rodriguez. Limit theorems for a general stochastic rumour model. arXiv: math.PR/1003.4995, 2010a. Citado na pág. 19

E. Lebensztayn, F. P. Machado e P. M. Rodriguez. On the behaviour of a rumor process with random stifling. Environ. Modelling Software, Aceito para publicação, 2010b. Citado na pág. 7

C. Lefevre e P. Picard. Distribution of the final extent of a rumour process. J. Appl. Prob., 31: 244-249, 1994. Citado na pág. 2

D. P. Maki e M. Thompson. Mathematical Models and Applications. Prentice-Hall, Englewood Cliffs, 1973. Citado na pág. 1, 2, 6

M. Nekovee, Y. Moreno, G. Bianconi e M. Marsili. Theory of rumour spreading in complex social networks. Phys. A, 374:457-470, 2007. Citado na pág. 1

C. E. M. Pearce. On the exact solution of the general stochastic rumour. Math. Comput. Modelling, 31:289-298, 2000. Citado na pág. 2, 23

B. Pittel. On a daley-kendall model of random rumours. J. Appl. Probab., 27:14-27, 1990. Citado na pág. 2,5

S. Yu. Popov. Frogs and some other interacting random walks models. Discrete Math. Theor. Comput. Sci., AC:277-288, 2003. Citado na pág. 28

A. Rapoport. Spread of information through a population with socio-structural bias: I. assumption of transitivity. Bull. Math. Biophys., 15:523-533, 1953a. Citado na pág. 1

A. Rapoport. Spread of information through a population with socio-structural bias: Ii. various models with partial transitivity. Bull. Math. Biophys., 15:535-546, 1953b. Citado na pág. 1

A. Rapoport e L. I. Rebhun. On the mathematical theory of rumour spread. Bull. Math. Biophys., 14:375-383, 1952. Citado na pág. 1

H. C. Schramm. An analytic framework for the war of ideas. Master's thesis, Naval Postgraduate School, Monterey, California, Estados Unidos, 2006. Citado na pág. 1, 5

A. Sudbury. The proportion of the population never hearing a rumour. J. Appl. Prob., 22: 443-446, 1985. Citado na pág. 2, 6, 14, 22

R. Watson. On the size of a rumour. Stoch. Proc. Appl., 27:141-149, 1988. Citado na pág. 2, 5, 6, 14,22 


\section{Índice Remissivo}

cadeia de Markov

"density dependent", 15, 24, 34

caracterização, 34

Lei dos Grandes Números, 35

Teorema Central do Limite, 36

geral, 20

reduzida, 15

contidos, 4

função

drift, 16, 25

derivadas parciais, 18, 26

de Lambert, 10

ramo inferior, 10, 21

ramo principal, 10, 21

grafo completo, 28-30

ignorantes, 4

proporção de

Lei dos Grandes Números, 10, 22

Teorema Central do Limite, 12, 22

informantes, 4

limite determinístico, 15, 24

modelo epidemico

SIR, 2

modelos de passeios aleatórios

modelo [ALMM], 28

modelo auxiliar, 29, 31

variante $k$-vidas, 28,31

sapos, 28

modelos de rumores

Daley-Kendall (ou [DK]), 4, 20, 22

variante $(\alpha, p), 5,20$

variante $(\alpha, p, q), 19,20,23$

variante $k$-fold stifling, 5

geral, 20

Hayes, 20, 22

história, 1
Kawachi, 20, 23

Maki-Thompson (ou [MT]), 6, 13, 20, 22

variante $k$-fold stifling, 6, 13, 28, 30, 31

variante random stifling, 7,13

Pearce, 20, 23

mudança do tempo, 15, 24

neutros, 19

proporção de

Lei dos Grandes Números, 22

Teorema Central do Limite, 22

particula, 28

ativa, 28,29

inativa, 28,29

passeio aleatório, 28, 29, 31

probabilidades de transição

para cadeias de Markov "density dependet", 34

para o modelo [DK], 4 variante $(\alpha, p), 5$

para o modelo [MT], 6

para o modelo SIR, 2

redução da dimensão, 15

tempo de absorção, 9, 20

transições

para cadeias de Markov "density dependent", 34

para o modelo [ALMM]

modelo auxiliar, 29

para o modelo [DK], 4

variante $(\alpha, p), 5$

variante $(\alpha, p, q), 19$

para o modelo [MT], 6

variante $k$-fold stifling, 30

variante random stifling, 8

para o modelo de rumor geral, 20

para o modelo SIR, 2 